\title{
IMPLICIT COUPLING OF ONE-DIMENSIONAL AND THREE-DIMENSIONAL BLOOD FLOW MODELS WITH COMPLIANT VESSELS*
}

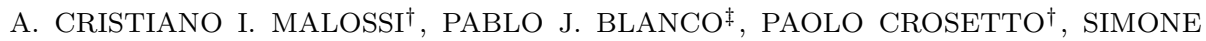 \\ DEPARIS $^{\dagger}$, AND ALFIO QUARTERONI ${ }^{\S}$
}

\begin{abstract}
The blood flow in arterial trees in the cardiovascular system can be simulated with the help of different models, depending on the outputs of interest and the desired degree of accuracy. In particular, one-dimensional fluid-structure interaction models for arteries are very effective in reproducing physiological pressure wave propagation and in providing quantities like pressure and velocity, averaged on the cross section of the arterial lumen. In locations where onedimensional models cannot capture the complete flow dynamics, e.g., in the presence of stenoses and aneurysms or other strong geometric perturbations, three-dimensional coupled fluid-structure interaction models are necessary to evaluate more accurately, for instance, critical factors responsible for pathologies which are associated with hemodynamics, such as wall shear stress. In this work we formalize and investigate the geometrical multiscale problem, where heterogeneous fluid-structure interaction models for arteries are implicitly coupled. We introduce new coupling algorithms, describe their implementation, and investigate on simple geometries the numerical reflections that occur at the interface between the heterogeneous models. We also simulate on a supercomputer a threedimensional abdominal aorta under physiological conditions, coupled with up to six one-dimensional models representing the surrounding arterial branches. Finally, we compare CPU times and number of coupling iterations for different algorithms and time discretizations.
\end{abstract}

Key words. blood flow models, geometrical multiscale modeling, coupling conditions, fluidstructure interaction, wave propagation

AMS subject classifications. 65M60, 74F10, 76D05, 92C35

DOI. $10.1137 / 120867408$

1. Introduction. Research in the field of hemodynamics is essential in order to understand, predict, and treat very common and dangerous cardiovascular pathologies, such as aneurysm formation, atherosclerosis, and congenital defects. Numerical simulations of blood flow dynamics are constantly improving in terms of reliability, efficiency, and model complexity. In this work we address the problem of accurately and efficiently simulating blood flow in the large arteries of the systemic circulation.

Since some of the main indicators for vascular pathologies are consequences of the vessel wall compliance (e.g., the pulse wave velocity), the deformation of the vessel

${ }^{*}$ Received by the editors February 24, 2012; accepted for publication (in revised form) February 25, 2013; published electronically April 30, 2013. The work of the first, third, fourth, and fifth authors was partly supported by the European Research Council Advanced grant "Mathcard, Mathematical Modelling and Simulation of the Cardiovascular System," Project ERC-2008-AdG 227058, and by the Swiss Platform for High-Performance and High-Productivity Computing (HP2C). This work was also supported by the Brazilian-Swiss Joint Research Programme (BSJRP), Project BJRP 011010 (N 590002/2010-4).

http://www.siam.org/journals/mms/11-2/86740.html

${ }^{\dagger}$ Chair of Modelling and Scientific Computing (CMCS), Mathematics Institute of Computational Science and Engineering (MATHICSE), École Polytechnique Fédérale de Lausanne (EPFL), Station 8, CH-1015 Lausanne, Switzerland (cristiano.malossi@epfl.ch, paolo.crosetto@epfl.ch, simone. deparis@epfl.ch).

${ }^{\ddagger}$ Laboratório Nacional de Computação Científica (LNCC), Quitandinha, 25651-075 Petrópolis, Brazil, and Instituto Nacional de Ciência e Tecnologia em Medicina Assistida por Computação Científica (INCT-MACC), Petrópolis, Brazil (pjblanco@lncc.br). This author's work was supported the Brazilian agencies CNPq and FAPERJ.

$\S$ MOX, Modeling and Scientific Computing, Department of Mathematics, Politecnico di Milano, Milan, Italy (alfio.quarteroni@epfl.ch). 
has to be taken into account to obtain reliable simulations and to draw meaningful conclusions. Modeling the corresponding fluid-structure interaction (FSI) can be achieved in several ways, e.g., using a one-dimensional (1-D) model that integrates the blood and the arterial wall (see, e.g., [4, 6, 17, 42]); performing a three-dimensional (3-D) simulation for the fluid inside the deformable lumen, where the deformation of the wall is accounted for at the variational level by a proper boundary condition on the endothelial wall (see, e.g., $[26,35,44]$ ); or considering the full interaction between the 3-D blood flow and a 3-D vessel wall by coupling the equations for the fluid flow with those for a solid structure (see $[2,10,12,27,33,43]$ and the references therein).

Since the time constraint is important in a medical environment, a compromise between model complexity and computational cost is mandatory. For this reason only a few specific arterial regions of the system are represented by 3 -D models, while the remaining parts are in general accounted for through reduced models. More precisely, 1-D models are used to represent the network of arteries (see, e.g., [31, 41]), while the heart and its valves, the veins, and the peripheral circulation are in general accounted for through lumped models (see $[21,36]$ and the references therein). The coupling of these heterogeneous models is what we call the geometrical multiscale problem. Its efficient solution is a challenging task which has been addressed by several research groups with the final goal of accounting for the whole circulation and autoregulation (see, e.g., $[5,18,24,26]$ ). The identification of the most convenient model representation is, evidently, problem-dependent. For example, when studying hemodynamics in cerebral aneurysms, it is necessary to employ 3-D representations of such geometrical perturbations to accurately model blood flow in the intra-aneurysmal region, but at the same time it is interesting, and in some cases mandatory, to consider the effect of the surrounding upstream and downstream vasculature through 1-D models. Other target applications are related to the performance of the cardiac muscle. In such situations, a 3-D model of the heart is needed to understand, for instance, the impact of arterial stiffening on the growth and remodeling of the left ventricle. This analysis can be done by coupling a 1-D model of the entire vasculature to the aortic valve. A third application we could think of is the study of tumor blood supply, in which, due to the high tortuosity and complex interconnectivity of blood vessels, a 3 -D simulation may be required to characterize the oxygen supply to the tumor cells, as well as the interaction of tumor cells with the blood flow to understand the mechanisms that are responsible for their migration to other parts of the body through the arterial circulation. In such a case, the 3-D model of the tumor blood vessels could be coupled to 1-D models representing the proximal healthy vasculature, the latter feeding the 3-D model of the tumor blood flow with an adequate hemodynamic environment according to the location within the human body.

In this work we propose different methods to handle the coupling of the heterogeneous FSI models. More precisely, first we address the coupling of the fluid part of the problem, which can be set up by imposing different types of quantities at the coupling interfaces [32]. Then we focus on the solid part: here, the 3-D solid problem requires an additional set of boundary conditions on the interface rings, while the 1-D problem is already closed thanks to the local relation between the pressure and the area of the vessel. To close the 3-D solid problem we propose several approaches. Among them, we also devise a technique to impose the continuity of the area with the surrounding reduced models, extending the work done in [19]. These conditions are often neglected in the literature, also due to their nontrivial implementation.

One of the main concerns when dealing with geometrical multiscale problems is 
the need to avoid, or reduce, numerical spurious reflections at the interface separating the two models (see $[3,15]$ ). Therefore, we study the influence of the proposed interface conditions on the amplitude of the spurious backward reflections that eventually arise at the coupling interfaces between two dimensionally heterogeneous pipes. In order to have a full overview of the phenomenon, we analyze both the case of a 1-D wave flow that enters in a 3-D pipe, and the inverse one, i.e., a 3-D wave flow that enters in a 1-D pipe. The former case is considered here for the first time, to the best of our knowledge. In fact, our goal is not only to devise a set of boundary conditions that absorb the numerical reflections in a 3-D simulation, but more generally to embed 3-D pieces in a 1-D network in order to be able to perform 3-D simulations within a consistent hemodynamics environment, in terms of upstream and downstream vascular impedances, provided by a proper 1-D model.

In addition, we also study the computational efficiency of the solution of the global coupled problem. Into a network composed of 1-D models we insert a real 3-D geometry of the abdominal aorta. This results in six coupling interfaces between 1-D FSI models and one 3-D FSI model. For this physiological test case we consider two different time discretizations for the 3-D FSI problem and two different methods for solving the coupling between the models. We compare the CPU time for all these options, and we investigate its dependence on the number of 1-D pieces considered for the coupling.

The methodology described in this work for the coupling of the heterogeneous models has been implemented as part of the C++ finite elements library LifeV [29], which is distributed under LGPL licence. Efficient and parallel solvers for 3-D FSI $[10,11]$ and 1-D FSI [31] problems have been exploited and coupled within a single general and extensible framework. In addition, the parallel implementation allows us to tackle large problems and to profitably use scalable supercomputers. The results of this work have been later used in [22] for the study of the physical consistency of the coupling between 3-D and 1-D FSI models for cardiovascular applications.

This work is organized as follows. Section 2 presents the governing equations, in continuous and discrete forms, of the 3-D and 1-D FSI models. Section 3 describes the fluid interface equations and the numerical algorithms used to solve the heterogeneous network of FSI problems. The interface conditions for the solid part of the FSI problems are addressed in section 4; more precisely, three different sets of boundary conditions are proposed to close the 3 -D solid problem. These conditions are then used and tested in section 5 on some benchmark problems, with the aim of analyzing and comparing the number of iterations and the impact of the spurious backward reflections at the coupling interfaces. In section 6 the performance of the different numerical techniques is analyzed in the context of a physiological scenario. Finally, our conclusions are reported in section 7 .

2. Fluid-structure interaction models. As previously stated, the interaction between blood flow and arterial wall deformation (compression and dilatation) has to be taken into account to correctly reconstruct the behavior of the arterial pulse. In fact, blood flow is characterized by traveling waves, generated by the interaction between the fluid pressure and the wall deformation. In this section we recall the 3 -D and the 1-D FSI models for the cardiovascular setting. For both models we then provide the numerical approximation techniques employed to solve the problems at the discrete level.

2.1. 3-D FSI model. In a geometrical multiscale setting, 3-D FSI models are used to simulate the hemodynamics in complex geometrical situations such as those 

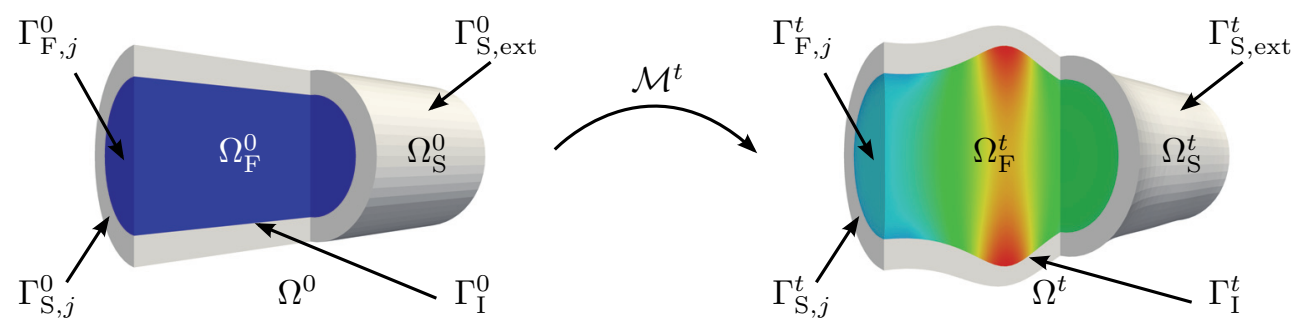

FIG. 2.1. Reference and current configurations with ALE mapping. The color map in the scheme indicates the computed blood pressure field.

occurring at bifurcations, aneurysms, and stenoses, among others. In addition, when aiming at patient-specific analyses, the correct characterization of the local arterial flow has to be carried out by using patient-specific data obtained from 3-D medical images.

2.1.1. Equations. Despite the complexity of blood rheology, a Newtonian incompressible fluid is a suitable model for blood at the scale of larger arteries [21]. The arterial blood flow is therefore modeled with the incompressible Navier-Stokes equations in the case of a moving fluid domain, resulting in the so-called arbitrary Lagrangian Eulerian (ALE) formulation [20]. This approach is motivated by the need to impose the boundary conditions for the fluid equations on a moving domain.

Let $\Omega^{0} \subset \mathbb{R}^{3}$ be a 3-D bounded domain with Lipschitz continuous boundary $\partial \Omega^{0}$, where $\bar{\Omega}^{0}=\bar{\Omega}_{\mathrm{F}}^{0} \cup \bar{\Omega}_{\mathrm{S}}^{0}$ denotes the reference configuration of the fluid-solid problem, $\Omega_{\mathrm{F}}^{0}$ and $\Omega_{\mathrm{S}}^{0}$ being the fluid and solid reference domains, respectively (see Figure 2.1). In addition, we denote by $\Gamma_{\mathrm{I}}^{0}$ the reference fluid-solid interface $\partial \Omega_{\mathrm{F}}^{0} \cap \partial \Omega_{\mathrm{S}}^{0}$. The ALE formulation leads to an arbitrary choice of the geometrical problem. In this work we describe the fluid domain displacement $\boldsymbol{d}_{\mathrm{F}}$ as the harmonic extension of the solid displacement $\boldsymbol{d}_{\mathrm{S}}$ at the fluid-solid interface $\Gamma_{\mathrm{I}}^{0}$ to the interior of the fluid reference domain $\Omega_{\mathrm{F}}^{0}$, i.e.,

$$
\left\{\begin{aligned}
-\boldsymbol{\Delta} \boldsymbol{d}_{\mathrm{F}}=\mathbf{0} & \text { in } \Omega_{\mathrm{F}}^{0}, \\
\boldsymbol{d}_{\mathrm{F}}-\boldsymbol{d}_{\mathrm{S}}=\mathbf{0} & \text { on } \Gamma_{\mathrm{I}}^{0}, \\
\boldsymbol{\nabla} \boldsymbol{d}_{\mathrm{F}} \cdot \boldsymbol{n}_{\mathrm{F}}=\mathbf{0} & \text { on } \partial \Omega_{\mathrm{F}}^{0} \backslash \Gamma_{\mathrm{I}}^{0},
\end{aligned}\right.
$$

with $\boldsymbol{n}_{\mathrm{F}}$ being the outgoing normal direction on the fluid domain boundary. The solid displacement $\boldsymbol{d}_{\mathrm{S}}$ changes with time, and therefore the harmonic extension (2.1) defines the current configuration of the fluid domain $\Omega_{\mathrm{F}}^{t}$, which is parametrized by the ALE map

$$
\begin{aligned}
\mathcal{M}^{t}: \Omega_{\mathrm{F}}^{0} & \rightarrow \Omega_{\mathrm{F}}^{t} \subset \mathbb{R}^{3}, \\
\boldsymbol{x}^{0} & \mapsto \mathcal{M}^{t}\left(\boldsymbol{x}^{0}\right)=\boldsymbol{x}^{0}+\boldsymbol{d}_{\mathrm{F}}\left(\boldsymbol{x}^{0}\right),
\end{aligned}
$$

such that $\Omega_{\mathrm{F}}^{t}=\mathcal{M}^{t}\left(\Omega_{\mathrm{F}}^{0}\right)$, with $\boldsymbol{x}^{0} \in \Omega_{\mathrm{F}}^{0}$ a fluid point in the reference configuration. This approach is very convenient in the description of deforming arteries, since it allows us to preserve the mesh topology during the simulations, just moving its vertices.

The fluid problem is modeled by the incompressible Navier-Stokes equations writ- 
ten in the ALE formulation

$$
\left\{\begin{aligned}
\rho_{\mathrm{F}}\left(\left.\frac{\partial \boldsymbol{u}_{\mathrm{F}}}{\partial t}\right|_{\boldsymbol{x}^{0}}+\left(\left(\boldsymbol{u}_{\mathrm{F}}-\boldsymbol{w}_{\mathrm{F}}\right) \cdot \boldsymbol{\nabla}\right) \boldsymbol{u}_{\mathrm{F}}\right)-\boldsymbol{\nabla} \cdot \sigma_{\mathrm{F}}=\mathbf{0} & \text { in } \Omega_{\mathrm{F}}^{t} \times(0, T], \\
\boldsymbol{\nabla} \cdot \boldsymbol{u}_{\mathrm{F}}=0 & \text { in } \Omega_{\mathrm{F}}^{t} \times(0, T], \\
\boldsymbol{u}_{\mathrm{F}}-\overline{\boldsymbol{u}}_{\mathrm{F}}=\mathbf{0} & \text { in } \Omega_{\mathrm{F}}^{t} \times\{0\},
\end{aligned}\right.
$$

where $(0, T]$ is the time interval, $\rho_{\mathrm{F}}$ the fluid density, $\sigma_{\mathrm{F}}=-p_{\mathrm{F}} \mathrm{I}+2 \mu_{\mathrm{F}} \epsilon_{\mathrm{F}}\left(\boldsymbol{u}_{\mathrm{F}}\right)$ the Cauchy stress tensor (with I the identity matrix), $\epsilon_{\mathrm{F}}\left(\boldsymbol{u}_{\mathrm{F}}\right)=\left(\boldsymbol{\nabla} \boldsymbol{u}_{\mathrm{F}}+\left(\boldsymbol{\nabla} \boldsymbol{u}_{\mathrm{F}}\right)^{\top}\right) / 2$ the strain rate tensor, $\boldsymbol{u}_{\mathrm{F}}$ the fluid velocity vector field, $\overline{\boldsymbol{u}}_{\mathrm{F}}$ the initial fluid velocity vector field, $p_{\mathrm{F}}$ the fluid hydrostatic pressure, $\mu_{\mathrm{F}}$ the given fluid dynamic viscosity, and $\boldsymbol{w}_{\mathrm{F}}$ the fluid domain velocity, which is defined as

$$
\boldsymbol{w}_{\mathrm{F}}=\left.\frac{\partial \mathcal{M}^{t}}{\partial t}\right|_{\boldsymbol{x}^{0}}=\left.\frac{\partial \boldsymbol{d}_{\mathrm{F}}}{\partial t}\right|_{\boldsymbol{x}^{0}}
$$

Problem (2.2) is closed by imposing an interface condition with the solid problem on $\Gamma_{\mathrm{I}}^{0}$ and inflow and outflow boundary conditions on $\Gamma_{\mathrm{F}, j}^{t} \subset \partial \Omega_{\mathrm{F}}^{t} \backslash \Gamma_{\mathrm{I}}^{t}, j=1, \ldots, n_{\mathrm{FS}}^{\Gamma}$. In a geometrical multiscale setting, some of these inflow/outflow boundaries are coupling interfaces with the nearby reduced dimensional model, where interface equations are imposed, as we discuss in section 3. On the remaining boundaries we can impose given data, such as inlet/outlet time flow/velocity profiles. Note that, for the sake of simplicity and without a loss of generality, we assume that the number of boundaries $n_{\mathrm{FS}}^{\Gamma}$ is the same for both the fluid and the solid problems, and hence we use the double subscript FS.

The solid problem is described in a purely Lagrangian frame of reference. A large variety of materials can be chosen for modeling the arterial wall. The mechanical response of the wall to a given strain is mainly due to the elastin and collagen components. The former is responsible for the isotropic hyperelastic response, while the latter incorporates anisotropy and constitutive nonlinearity into the strain-stress relation; it is activated only when the strains reach a certain critical value and in certain directions (according to fiber orientation). In this regard, we consider a linear elastic model for the elastin and neglect the nonlinearities due to fiber orientation and activation. In addition, we assume a quasi-incompressible behavior for the arterial tissue and neglect its viscoelastic response. In fact, an equivalent viscoelastic effect is accounted for through proper boundary conditions acting over the external wall surface coming from a model of the surrounding tissues (see, e.g., [34]). As a result of these considerations, in this work we consider a linear elastic isotropic St. Venant-Kirchhoff model to describe the solid displacement:

$$
\left\{\begin{aligned}
\rho_{\mathrm{S}} \frac{\partial^{2} \boldsymbol{d}_{\mathrm{S}}}{\partial t^{2}}-\boldsymbol{\nabla} \cdot \sigma_{\mathrm{S}}=\mathbf{0} & \text { in } \Omega_{\mathrm{S}}^{0} \times(0, T] \\
\boldsymbol{d}_{\mathrm{S}}-\overline{\boldsymbol{d}}_{\mathrm{S}}=\mathbf{0} & \text { in } \Omega_{\mathrm{S}}^{0} \times\{0\} \\
\frac{\partial \boldsymbol{d}_{\mathrm{S}}}{\partial t}-\overline{\boldsymbol{v}}_{\mathrm{S}}=\mathbf{0} & \text { in } \Omega_{\mathrm{S}}^{0} \times\{0\}
\end{aligned}\right.
$$

where $\rho_{\mathrm{S}}$ is the solid density; $\sigma_{\mathrm{S}}=\lambda_{\mathrm{S}} \operatorname{tr}\left(\epsilon_{\mathrm{S}}\left(\boldsymbol{d}_{\mathrm{S}}\right)\right) \mathrm{I}+2 \mu_{\mathrm{S}} \epsilon_{\mathrm{S}}\left(\boldsymbol{d}_{\mathrm{S}}\right)$ the first Piola-Kirchhoff stress tensor, where $\epsilon_{\mathrm{S}}\left(\boldsymbol{d}_{\mathrm{S}}\right)=\left(\boldsymbol{\nabla} \boldsymbol{d}_{\mathrm{S}}+\left(\boldsymbol{\nabla} \boldsymbol{d}_{\mathrm{S}}\right)^{\top}\right) / 2 ; \lambda_{\mathrm{S}}$ and $\mu_{\mathrm{S}}$ are the first and second Lamé parameters, respectively; $\overline{\boldsymbol{d}}_{\mathrm{S}}$ is the initial solid displacement; and $\overline{\boldsymbol{v}}_{\mathrm{S}}$ the initial solid velocity. The St. Venant-Kirchhoff materials are usually characterized by the 
Young's modulus $E_{\mathrm{S}}$ and the Poisson ratio $\nu_{\mathrm{S}}$. The following relations hold between the two sets of coefficients:

$$
\lambda_{\mathrm{S}}=\frac{E_{\mathrm{S}} \nu_{\mathrm{S}}}{\left(1-2 \nu_{\mathrm{S}}\right)\left(1+\nu_{\mathrm{S}}\right)}, \quad \mu_{\mathrm{S}}=\frac{E_{\mathrm{S}}}{2\left(1+\nu_{\mathrm{S}}\right)} .
$$

Problem (2.3) is closed by imposing an interface condition with the fluid problem on $\Gamma_{\mathrm{I}}^{0}$ and a proper set of boundary conditions on $\partial \Omega_{\mathrm{S}}^{0} \backslash \Gamma_{\mathrm{I}}^{0}$. On the solid external wall $\Gamma_{\mathrm{S}, \text { ext }}^{0}$ we can apply either a Neumann boundary condition, to account for a distributed pressure field, or a viscoelastic Robin boundary condition, to model the presence of the external tissues on the arteries. In turn, in a geometrical multiscale setting, the area of the $n_{\mathrm{FS}}^{\Gamma}$ inlet/outlet solid rings $\Gamma_{\mathrm{S}, j}^{0}, j=1, \ldots, n_{\mathrm{FS}}^{\Gamma}$, can be related to that of the surrounding models; in contrast, if there exists any uncoupled boundary interface, a different approach must be followed. We defer this discussion to section 4 .

2.1.2. Solution strategy for the FSI problem. The solution of the FSI problem can be obtained by using either a modular (segregated) or nonmodular (monolithic) strategy. Depending on the choice of the boundary conditions applied to the fluid and solid domains, the former approach may lead to Dirichlet-Neumann [12, 13], Neumann-Dirichlet [8], Robin-Neumann [1], or Robin-Robin [23] approaches. In all cases, the main drawback is that an additional subiteration algorithm is required to achieve strong coupling between the fluid and the solid problems. To avoid fluid-solid subiterations, we use a nonmodular strategy, where the fluid and the solid are treated as a single problem. The solution algorithm relies on the parallel block preconditioner developed in [11], which is based on an inexact block factorization, where each factor is indeed an algebraic additive Schwarz preconditioner. The resulting preconditioner has proven to be effective and scalable, since each different factor addresses a single subproblem in a specific way.

Problems (2.2) and (2.3) are coupled through interface conditions on $\Gamma_{\mathrm{I}}^{0}$, which comprise the continuity of the velocity field and that of the traction (the latter with the use of a Lagrange multiplier). The interface problem reads

$$
\left\{\begin{aligned}
\boldsymbol{u}_{\mathrm{F}} \circ \mathcal{M}^{t}-\frac{\partial \boldsymbol{d}_{\mathrm{S}}}{\partial t}=\mathbf{0} & & \text { on } \Gamma_{\mathrm{I}}^{0} \times(0, T], \\
\sigma_{\mathrm{S}} \cdot \boldsymbol{n}_{\mathrm{S}}-\boldsymbol{\lambda}_{\Gamma_{\mathrm{I}}}=\mathbf{0} & & \text { on } \Gamma_{\mathrm{I}}^{0} \times(0, T], \\
\boldsymbol{\lambda}_{\Gamma_{\mathrm{I}}}-J_{\mathrm{S}} \mathrm{G}_{\mathrm{S}}^{-\mathrm{T}}\left(\sigma_{\mathrm{F}} \circ \mathcal{M}^{t}\right) \cdot \boldsymbol{n}_{\mathrm{S}}=\mathbf{0} & & \text { on } \Gamma_{\mathrm{I}}^{0} \times(0, T],
\end{aligned}\right.
$$

where $\boldsymbol{\lambda}_{\Gamma_{\mathrm{I}}}$ is the Lagrange multiplier, $\boldsymbol{n}_{\mathrm{S}}$ the outgoing normal direction on the solid domain, $\mathrm{G}_{\mathrm{S}}=\mathrm{I}+\boldsymbol{\nabla} \boldsymbol{d}_{\mathrm{S}}$ the solid deformation gradient, and $J_{\mathrm{S}}=\operatorname{det}\left(\mathrm{G}_{\mathrm{S}}\right)$. For ease of writing, let us formally denote problems (2.1), (2.2), (2.3), and (2.4) in their weak form as $\mathbf{G}, \mathbf{F}, \mathbf{S}$, and $\mathbf{I}$, respectively. This allows us to write the global variational formulation of the FSI problem in a compact form as follows: find $\left(\boldsymbol{u}_{\mathrm{F}}, p_{\mathrm{F}}\right) \in \mathcal{V}_{\mathrm{F}}$, $\boldsymbol{d}_{\mathrm{S}} \in \mathcal{V}_{\mathrm{S}}, \lambda_{\Gamma_{\mathrm{I}}} \in \mathcal{V}_{\mathrm{I}}$, and $\boldsymbol{d}_{\mathrm{F}} \in \mathcal{V}_{\mathrm{G}}$ such that

$$
\left\{\begin{aligned}
\mathbf{F}\left(\left(\boldsymbol{u}_{\mathrm{F}}, p_{\mathrm{F}}, \boldsymbol{\lambda}_{\Gamma_{\mathrm{I}}}, \boldsymbol{d}_{\mathrm{F}}\right),\left(\boldsymbol{u}_{\mathrm{F}}^{*}, p_{\mathrm{F}}^{*}\right)\right)=\mathbf{0} & \forall\left(\boldsymbol{u}_{\mathrm{F}}^{*}, p_{\mathrm{F}}^{*}\right) \in \mathcal{V}_{\mathrm{F}}^{*} \\
\mathbf{S}\left(\left(\boldsymbol{d}_{\mathrm{S}}, \boldsymbol{\lambda}_{\Gamma_{\mathrm{I}}}\right), \boldsymbol{d}_{\mathrm{S}}^{*}\right)=\mathbf{0} & \forall \boldsymbol{d}_{\mathrm{S}}^{*} \in \mathcal{V}_{\mathrm{S}}^{*} \\
\mathbf{I}\left(\left(\boldsymbol{u}_{\mathrm{F}}, \boldsymbol{d}_{\mathrm{S}}\right), \boldsymbol{\lambda}_{\Gamma_{\mathrm{I}}}^{*}\right)=\mathbf{0} & \forall \boldsymbol{\lambda}_{\Gamma_{\mathrm{I}}}^{*} \in \mathcal{V}_{\mathrm{I}}^{*} \\
\mathbf{G}\left(\left(\boldsymbol{d}_{\mathrm{S}}, \boldsymbol{d}_{\mathrm{F}}\right), \boldsymbol{d}_{\mathrm{F}}^{*}\right)=\mathbf{0} & \forall \boldsymbol{d}_{\mathrm{F}}^{*} \in \mathcal{V}_{\mathrm{G}}^{*}
\end{aligned}\right.
$$

$\mathcal{V}_{\mathrm{F}}, \mathcal{V}_{\mathrm{S}}, \mathcal{V}_{\mathrm{I}}$, and $\mathcal{V}_{\mathrm{G}}$ are proper linear manifolds including the corresponding essential conditions according to the boundary data; the superscript $*$ identifies test functions and spaces. 
2.1.3. Numerical approximation. The fluid problem is discretized in space by a $\mathbb{P} 1-\mathbb{P} 1$ finite element method, stabilized by an interior penalty technique [7]. The solid and the geometric problems are discretized in space by $\mathbb{P} 1$ finite elements. These discretizations show a first order convergence in space. A large variety of time discretizations for the incompressible Navier-Stokes equations on moving domains can be found in the literature, depending on the target application. In this work we use a first order Euler scheme. Regarding the solid problem, we use a second order midpoint scheme, which can be interpreted as a Newmark scheme with a particular choice of the coefficients. The extension of the proposed methodology to other space and time discretizations is straightforward and not a matter of concern here. The global time interval $[0, T]$ is split into several uniform subintervals $\left[t^{n}, t^{n+1}\right], n=0,1,2, \ldots$, such that $t^{n}=n \Delta t$, with $\Delta t$ being the time step. Regarding the notation in the following we use the superscripts $n$ and $n+1$ to refer to quantities at time $t^{n}$ and $t^{n+1}$, respectively, and the $h$ subscript for quantities discretized in space.

Problem (2.5) is highly nonlinear due to the Navier-Stokes convective term and to the displacement of the fluid domain. Following [11], we use two time discretization strategies to address such nonlinearities. In both cases, the solution of the nonmodular FSI problem is performed by using the Newton method [10, 11]. Several approximations of the corresponding Jacobian matrix by finite difference methods are reviewed in the literature (see, e.g., $[27,33,43]$ ). However, these schemes may lead to a substantial increase of the number of iterations required for the convergence to the solution of the FSI problem. For this reason, in this work we compute the Jacobian blocks by an analytic formulation, which takes into account the shape derivatives, i.e., the cross derivatives of the fluid problem with respect to the domain motion. For a detailed description, including the derivation of the Jacobian blocks, see $[10$, section 3.4] and [14].

Let $\boldsymbol{u}_{\mathrm{FSI}}$ be the vector of unknowns of the FSI problem. The $p$ th iteration of the Newton method reads

$$
\boldsymbol{u}_{\mathrm{FSI}, h}^{p+1}=\boldsymbol{u}_{\mathrm{FSI}, h}^{p}+\delta \boldsymbol{u}_{\mathrm{FSI}, h}^{p},
$$

where the update $\delta \boldsymbol{u}_{\mathrm{FSI}, h}^{p}$ is computed by solving

$$
\mathcal{J}_{\mathrm{FSI}}\left(\boldsymbol{u}_{\mathrm{FSI}, h}^{p}\right) \delta \boldsymbol{u}_{\mathrm{FSI}, h}^{p}=-\boldsymbol{\mathcal { R }}_{\mathrm{FSI}}\left(\boldsymbol{u}_{\mathrm{FSI}, h}^{p}\right)
$$

This requires the computation of the Jacobian matrix $\mathcal{J}_{\mathrm{FSI}}\left(\boldsymbol{u}_{\mathrm{FSI}, h}^{p}\right)$ and the evaluation of the residuals vector $\boldsymbol{\mathcal { R }}_{\mathrm{FSI}}\left(\boldsymbol{u}_{\mathrm{FSI}, h}^{p}\right)$ at each iteration. The specific expression of these two elements depends on the chosen time discretization strategy.

Fully implicit (FI) time discretization. In the FI time discretization scheme, the geometric and convective terms in the equations are considered implicitly, such that the unknowns vector is $\boldsymbol{u}_{\mathrm{FSI}, h}=\left(\boldsymbol{y}_{\mathrm{F}, h}^{\top}, \boldsymbol{d}_{\mathrm{S}, h}^{\top}, \boldsymbol{\lambda}_{\Gamma_{\mathrm{I}}, h}^{\top}, \boldsymbol{d}_{\mathrm{F}, h}^{\top}\right)^{\top}$, with $\boldsymbol{y}_{\mathrm{F}, h}=\left(\boldsymbol{u}_{\mathrm{F}, h}^{\top}, p_{\mathrm{F}, h}^{\top}\right)^{\top}$. The FI formulation reads: given $\boldsymbol{u}_{\mathrm{FSI}, h}^{n}$, find $\boldsymbol{u}_{\mathrm{FSI}, h}^{n+1}$ such that

$$
\mathrm{A}_{\mathrm{FSI}}\left(\boldsymbol{u}_{\mathrm{FSI}, h}^{n+1}\right) \boldsymbol{u}_{\mathrm{FSI}, h}^{n+1}=\boldsymbol{b}_{\mathrm{FSI}}\left(\boldsymbol{u}_{\mathrm{FSI}, h}^{n+1}\right),
$$


where the finite element matrix $\mathrm{A}_{\mathrm{FSI}}$ depends on the solution vector $\boldsymbol{u}_{\mathrm{FSI}, h}^{n+1}$ and can be written in a block form as

$$
\begin{aligned}
& \mathrm{A}_{\mathrm{FSI}}\left(\boldsymbol{u}_{\mathrm{FSI}, h}^{n+1}\right) \\
& =\left[\begin{array}{cc|cc|c|cc}
\mathrm{F}_{\mathrm{FF}}\left(\boldsymbol{y}_{\mathrm{F}, h}^{n+1}, \boldsymbol{d}_{\mathrm{F}, h}^{n+1}\right) & \mathrm{F}_{\mathrm{F} \Gamma_{\mathrm{I}}}\left(\boldsymbol{y}_{\mathrm{F}, h}^{n+1}, \boldsymbol{d}_{\mathrm{F}, h}^{n+1}\right) & 0 & 0 & 0 & 0 & 0 \\
\mathrm{~F}_{\Gamma_{\mathrm{I}} \mathrm{F}}\left(\boldsymbol{y}_{\mathrm{F}, h}^{n+1}, \boldsymbol{d}_{\mathrm{F}, h}^{n+1}\right) & \mathrm{F}_{\Gamma_{\mathrm{I}} \Gamma_{\mathrm{I}}}\left(\boldsymbol{y}_{\mathrm{F}, h}^{n+1}, \boldsymbol{d}_{\mathrm{F}, h}^{n+1}\right) & 0 & 0 & \Lambda_{\mathrm{F}} & 0 & 0 \\
\hline 0 & 0 & \mathrm{~S}_{\mathrm{SS}} & \mathrm{S}_{\mathrm{S} \Gamma_{\mathrm{I}}} & 0 & 0 & 0 \\
0 & 0 & \mathrm{~S}_{\Gamma_{\mathrm{I}} \mathrm{S}} & \mathrm{S}_{\Gamma_{\mathrm{I}} \Gamma_{\mathrm{I}}} & -\mathrm{I} & 0 & 0 \\
\hline 0 & \Lambda_{\mathrm{F}}^{\mathrm{T}} & 0 & -\mathrm{I} / \Delta t & 0 & 0 & 0 \\
\hline 0 & 0 & 0 & 0 & \mathrm{G}_{\mathrm{FF}} & \mathrm{G}_{\mathrm{F} \Gamma_{\mathrm{I}}} \\
0 & 0 & 0 & -\mathrm{I} & 0 & 0 & \mathrm{I}
\end{array}\right],
\end{aligned}
$$

while

$$
\boldsymbol{u}_{\mathrm{FSI}, h}^{n+1}=\left(\begin{array}{c}
\boldsymbol{y}_{\mathrm{FF}, h}^{n+1} \\
\boldsymbol{y}_{\mathrm{F} \Gamma_{\mathrm{I}}, h}^{n+1} \\
\hline \boldsymbol{d}_{\mathrm{SS}, h}^{n+1} \\
\boldsymbol{d}_{\mathrm{S} \Gamma_{\mathrm{I}}, h}^{n+1} \\
\hline \lambda_{\Gamma_{\mathrm{I}}, h}^{n+1} \\
\hline \boldsymbol{d}_{\mathrm{FF}, h}^{n+1} \\
\boldsymbol{d}_{\mathrm{F} \Gamma_{\mathrm{I}}, h}^{n+1}
\end{array}\right), \quad \boldsymbol{b}_{\mathrm{FSI}}\left(\boldsymbol{u}_{\mathrm{FSI}, h}^{n+1}\right)=\left(\begin{array}{c}
\boldsymbol{b}_{\mathrm{FF}}\left(\boldsymbol{d}_{\mathrm{F}, h}^{n+1}\right) \\
\frac{\boldsymbol{b}_{\mathrm{F} \Gamma_{\mathrm{I}}}\left(\boldsymbol{d}_{\mathrm{F}, h}^{n+1}\right)}{\boldsymbol{b}_{\mathrm{SS}}} \\
\frac{\boldsymbol{b}_{\mathrm{S} \Gamma_{\mathrm{I}}}}{-\boldsymbol{d}_{\mathrm{S} \Gamma_{\mathrm{I}}, h}^{n} / \Delta t} \\
\mathbf{0} \\
\mathbf{0}
\end{array}\right) .
$$

In the algebraic form, for the sake of clarity, we distinguish between the degrees of freedom lying on the fluid-solid interface (adding the subscript $\Gamma_{I}$ to the blocks) and those that are internal to the domains $\Omega_{\mathrm{F}}^{t}$ and $\Omega_{\mathrm{S}}^{0}$ (for which we keep the same subscripts as for the continuous case). In particular, $\boldsymbol{y}_{\mathrm{F}, h}=\left(\boldsymbol{y}_{\mathrm{FF}, h}^{\top}, \boldsymbol{y}_{\mathrm{F} \Gamma_{\mathrm{I}}, h}^{\top}\right)^{\top}, \boldsymbol{d}_{\mathrm{S}}=\left(\boldsymbol{d}_{\mathrm{SS}, h}^{\top}, \boldsymbol{d}_{\mathrm{S} \Gamma_{\mathrm{I}}, h}^{\top}\right)^{\top}$, and $\boldsymbol{d}_{\mathrm{F}, h}=\left(\boldsymbol{d}_{\mathrm{FF}, h}^{\top}, \boldsymbol{d}_{\mathrm{F} \Gamma_{\mathrm{I}}, h}^{\top}\right)^{\top}$. Blocks $(1,1),(2,2)$, and $(4,4)$ represent the discrete fluid, solid, and geometric problems, respectively. The off-diagonal entries enforce the coupling conditions between the three fields. The rectangular block matrix $\Lambda_{\mathrm{F}}$ coincides with $[\mathrm{I}, 0]^{\top}$ such that it selects the fluid velocity degrees of freedom of $\boldsymbol{y}_{\mathrm{F}_{\mathrm{I}}, h}$ to apply the fluid-solid interface conditions. Note that in this discrete formulation we suppose a first order time discretization of the velocity continuity condition. The nonlinearities, i.e., the dependence of the fluid blocks from the fluid displacement and from the fluid velocity and pressure fields, are explicitly indicated in the matrix entries. The fluid and solid right-hand sides including the terms generated by the time discretization of the momentum conservation equations are denoted by $\boldsymbol{b}_{\mathrm{F}}=\left(\boldsymbol{b}_{\mathrm{FF}}^{\top}, \boldsymbol{b}_{\mathrm{F} \Gamma_{\mathrm{I}}}^{\top}\right)^{\top}$ and $\boldsymbol{b}_{\mathrm{S}}=\left(\boldsymbol{b}_{\mathrm{SS}}^{\top}, \boldsymbol{b}_{\mathrm{S} \Gamma_{\mathrm{I}}}^{\top}\right)^{\top}$, respectively. We remark that, due to the implicit formulation adopted for the fluid domain, also the fluid right-hand side depends on the fluid domain displacement.

The residual of the Newton method is defined as

$$
\boldsymbol{\mathcal { R }}_{\mathrm{FSI}}\left(\boldsymbol{u}_{\mathrm{FSI}, h}^{n+1}\right)=\mathrm{A}_{\mathrm{FSI}}\left(\boldsymbol{u}_{\mathrm{FSI}, h}^{n+1}\right) \boldsymbol{u}_{\mathrm{FSI}, h}^{n+1}-\boldsymbol{b}_{\mathrm{FSI}}\left(\boldsymbol{u}_{\mathrm{FSI}, h}^{n+1}\right),
$$


while the Jacobian matrix assumes the form

$\mathcal{J}_{\mathrm{FSI}}\left(\boldsymbol{u}_{\mathrm{FSI}, h}^{n+1}\right)=$
$\left[\begin{array}{cc|cc|c|cc}\mathrm{D}_{\boldsymbol{y}_{\mathrm{FF}}} \mathrm{F}_{\mathrm{F}} & \mathrm{D}_{\boldsymbol{y}_{\mathrm{F} \Gamma_{\mathrm{I}}}} \mathrm{F}_{\mathrm{F}} & 0 & 0 & 0 & \mathrm{D}_{\boldsymbol{d}_{\mathrm{FF}}}\left(\mathrm{F}_{\mathrm{F}}-\boldsymbol{b}_{\mathrm{F}}\right) & \mathrm{D}_{\boldsymbol{d}_{\mathrm{F} \Gamma_{\mathrm{I}}}}\left(\mathrm{F}_{\mathrm{F}}-\boldsymbol{b}_{\mathrm{F}}\right) \\ \mathrm{D}_{\boldsymbol{y}_{\mathrm{FF}}} \mathrm{F}_{\Gamma_{\mathrm{I}}} & \mathrm{D}_{\boldsymbol{y}_{\mathrm{F}_{\mathrm{I}}}} \mathrm{F}_{\Gamma_{\mathrm{I}}} & 0 & 0 & \Lambda_{\mathrm{F}} & \mathrm{D}_{\boldsymbol{d}_{\mathrm{FF}}}\left(\mathrm{F}_{\Gamma_{\mathrm{I}}}-\boldsymbol{b}_{\Gamma_{\mathrm{I}}}\right) & \mathrm{D}_{\boldsymbol{d}_{\mathrm{F} \Gamma_{\mathrm{I}}}}\left(\mathrm{F}_{\Gamma_{\mathrm{I}}}-\boldsymbol{b}_{\Gamma_{\mathrm{I}}}\right) \\ \hline 0 & 0 & \mathrm{~S}_{\mathrm{SS}} & \mathrm{S}_{\mathrm{S} \Gamma_{\mathrm{I}}} & 0 & 0 & 0 \\ 0 & 0 & \mathrm{~S}_{\Gamma_{\mathrm{I}} \mathrm{S}} & \mathrm{S}_{\Gamma_{\mathrm{I}} \Gamma_{\mathrm{I}}} & -\mathrm{I} & 0 & 0 \\ \hline 0 & \Lambda_{\mathrm{F}}^{\mathrm{T}} & 0 & -\mathrm{I} / \Delta t & 0 & 0 & 0 \\ \hline 0 & 0 & 0 & 0 & 0 & \mathrm{G}_{\mathrm{FF}} & \mathrm{G}_{\mathrm{F} \Gamma_{\mathrm{I}}} \\ 0 & 0 & 0 & -\mathrm{I} & 0 & 0 & \mathrm{I}\end{array}\right]$.

Note that in the Jacobian matrix we have used the abridged notation

$$
\begin{aligned}
& \mathrm{D}_{\mathbf{x}} \mathrm{F}_{\mathrm{F}}=\mathrm{D}_{\mathbf{x}} \mathrm{F}_{\mathrm{FF}}\left(\boldsymbol{y}_{\mathrm{F}, h}^{n+1}, \boldsymbol{d}_{\mathrm{F}, h}^{n+1}\right)+\mathrm{D}_{\mathbf{x}} \mathrm{F}_{\mathrm{F} \Gamma_{\mathrm{I}}}\left(\boldsymbol{y}_{\mathrm{F}, h}^{n+1}, \boldsymbol{d}_{\mathrm{F}, h}^{n+1}\right), \\
& \mathrm{D}_{\mathbf{x}} \mathrm{F}_{\Gamma_{\mathrm{I}}}=\mathrm{D}_{\mathbf{x}} \mathrm{F}_{\Gamma_{\mathrm{I}} \mathrm{F}}\left(\boldsymbol{y}_{\mathrm{F}, h}^{n+1}, \boldsymbol{d}_{\mathrm{F}, h}^{n+1}\right)+\mathrm{D}_{\mathbf{x}} \mathrm{F}_{\Gamma_{\mathrm{I}} \Gamma_{\mathrm{I}}}\left(\boldsymbol{y}_{\mathrm{F}, h}^{n+1}, \boldsymbol{d}_{\mathrm{F}, h}^{n+1}\right), \\
& \mathrm{D}_{\mathbf{x}} \boldsymbol{b}_{\mathrm{F}}=\mathrm{D}_{\mathbf{x}} \boldsymbol{b}_{\mathrm{FF}}\left(\boldsymbol{d}_{\mathrm{F}, h}^{n+1}\right)+\mathrm{D}_{\mathbf{x}} \boldsymbol{b}_{\mathrm{F} \Gamma_{\mathrm{I}}}\left(\boldsymbol{d}_{\mathrm{F}, h}^{n+1}\right), \\
& \mathrm{D}_{\mathbf{x}} \boldsymbol{b}_{\Gamma_{\mathrm{I}}}=\mathrm{D}_{\mathbf{x}} \boldsymbol{b}_{\Gamma_{\mathrm{I}} \mathrm{F}}\left(\boldsymbol{d}_{\mathrm{F}, h}^{n+1}\right)+\mathrm{D}_{\mathbf{x}} \boldsymbol{b}_{\Gamma_{\mathrm{I}} \Gamma_{\mathrm{I}}}\left(\boldsymbol{d}_{\mathrm{F}, h}^{n+1}\right),
\end{aligned}
$$

$D_{\mathbf{x}}$ being the total derivative of a given functional with respect to a variable $\mathbf{x}$.

Geometric convective explicit (GCE) time discretization. In the GCE time discretization scheme, at each new time iteration we define appropriate extrapolations $\boldsymbol{d}_{\mathrm{S} \Gamma_{\mathrm{I}}, h}^{\diamond}$ and $\boldsymbol{u}_{\mathrm{F}, h}^{\diamond}$ of the geometric and convective terms, respectively. In particular, we choose the simple approximation $\boldsymbol{d}_{\mathrm{S} \Gamma_{\mathrm{I}}, h}^{\diamond}=\boldsymbol{d}_{\mathrm{S} \Gamma_{\mathrm{I}}, h}^{n}$ and $\boldsymbol{u}_{\mathrm{F}, h}^{\diamond}=\boldsymbol{u}_{\mathrm{F}, h}^{n}$ such that the geometric and the fluid-solid parts of the problem can then be split. Hence, at each time step, first we compute the fluid displacement $\boldsymbol{d}_{\mathrm{F}, h}^{n+1}$ by solving the linear system associated with the geometric problem, i.e.,

$$
\left[\begin{array}{cc}
\mathrm{G}_{\mathrm{FF}} & \mathrm{G}_{\mathrm{F} \Gamma_{\mathrm{I}}} \\
0 & \mathrm{I}
\end{array}\right]\left(\begin{array}{c}
\boldsymbol{d}_{\mathrm{FF}, h}^{n+1} \\
\boldsymbol{d}_{\mathrm{F} \Gamma_{\mathrm{I}}, h}^{n+1}
\end{array}\right)-\left(\begin{array}{c}
\mathbf{0} \\
\boldsymbol{d}_{\mathrm{S} \Gamma_{\mathrm{I}}}^{\diamond}
\end{array}\right)=\mathbf{0},
$$

and we move the fluid mesh accordingly. Then, by computing the resulting fluid domain velocity as

$$
\boldsymbol{w}_{\mathrm{F}, h}^{n+1}=\frac{\boldsymbol{d}_{\mathrm{F}, h}^{n+1}-\boldsymbol{d}_{\mathrm{F}, h}^{n}}{\Delta t}
$$

we can replace the convective term in the fluid momentum equation by the quantity $\left(\left(\boldsymbol{u}_{\mathrm{F}, h}^{\diamond}-\boldsymbol{w}_{\mathrm{F}, h}^{n+1}\right) \cdot \boldsymbol{\nabla}\right) \boldsymbol{u}_{\mathrm{F}, h}^{n+1}$. Hence, the unknowns vector is reduced to $\boldsymbol{u}_{\mathrm{FSI}, h}=$ $\left(\boldsymbol{y}_{\mathrm{F}, h}^{\top}, \boldsymbol{d}_{\mathrm{S}, h}^{\top}, \boldsymbol{\lambda}_{\Gamma_{\mathrm{I}}, h}^{\top}\right)^{\top}$, and the second step reads: given $\boldsymbol{u}_{\mathrm{FSI}, h}^{n}$, find $\boldsymbol{u}_{\mathrm{FSI}, h}^{n+1}$ such that

$$
\mathrm{A}_{\mathrm{FSI}} \boldsymbol{u}_{\mathrm{FSI}, h}^{n+1}=\boldsymbol{b}_{\mathrm{FSI}}
$$


where now the finite element matrix $A_{\mathrm{FSI}}$ does not depend on $\boldsymbol{u}_{\mathrm{FSI}, h}^{n+1}$ such that its block form reads

$$
\mathrm{A}_{\mathrm{FSI}}=\left[\begin{array}{cc|cc|c}
\mathrm{F}_{\mathrm{FF}} & \mathrm{F}_{\mathrm{F} \Gamma_{\mathrm{I}}} & 0 & 0 & 0 \\
\mathrm{~F}_{\Gamma_{\mathrm{I}} \mathrm{F}} & \mathrm{F}_{\Gamma_{I} \Gamma_{\mathrm{I}}} & 0 & 0 & \Lambda_{\mathrm{F}} \\
\hline 0 & 0 & \mathrm{~S}_{\mathrm{SS}} & \mathrm{S}_{\mathrm{S} \Gamma_{I}} & 0 \\
0 & 0 & \mathrm{~S}_{\Gamma_{\mathrm{I}} \mathrm{S}} & \mathrm{S}_{\Gamma_{\mathrm{I}} \Gamma_{\mathrm{I}}} & -\mathrm{I} \\
\hline 0 & \Lambda_{\mathrm{F}}^{\mathrm{T}} & 0 & -\mathrm{I} / \Delta t & 0
\end{array}\right]
$$

while

$$
\boldsymbol{u}_{\mathrm{FSI}, h}^{n+1}=\left(\begin{array}{c}
\boldsymbol{y}_{\mathrm{FF}, h}^{n+1} \\
\boldsymbol{y}_{\mathrm{F} \Gamma_{\mathrm{I}}, h}^{n+1} \\
\hline \boldsymbol{d}_{\mathrm{SS}, h}^{n+1} \\
\boldsymbol{d}_{\mathrm{S} \Gamma_{\mathrm{I}}, h}^{n+1} \\
\hline \boldsymbol{\lambda}_{\Gamma_{\mathrm{I}}, h}^{n+1}
\end{array}\right), \quad \boldsymbol{b}_{\mathrm{FSI}}=\left(\begin{array}{c}
\boldsymbol{b}_{\mathrm{FF}} \\
\boldsymbol{b}_{\mathrm{F} \Gamma_{\mathrm{I}}} \\
\hline \boldsymbol{b}_{\mathrm{SS}} \\
\frac{\boldsymbol{b}_{\mathrm{S} \Gamma_{\mathrm{I}}}}{-\boldsymbol{d}_{\mathrm{S} \Gamma_{\mathrm{I}}}^{n} / \Delta t}
\end{array}\right)
$$

The residual of the Newton method is defined as

$$
\boldsymbol{\mathcal { R }}_{\mathrm{FSI}}\left(\boldsymbol{u}_{\mathrm{FSI}, h}^{n+1}\right)=\mathrm{A}_{\mathrm{FSI}} \boldsymbol{u}_{\mathrm{FSI}, h}^{n+1}-\boldsymbol{b}_{\mathrm{FSI}},
$$

and, since the problem is linear, the Jacobian matrix is equal to the system matrix, and the Newton method converges in one iteration.

2.2. 1-D FSI model. In a geometrical multiscale setting, the global arterial circulation can be modeled by a network of 1-D FSI segments, each characterized by a circular cross section (eventually narrowed along the axial direction) and a viscoelastic arterial wall (see, e.g., $[17,31,41]$ ). Despite the fact that the 1-D FSI model provides a simple axial symmetric representation of blood flow, it has proven to be able to accurately describe the behavior of the principal physiological quantities in a mean sense with respect to the transversal area of the vessel.

2.2.1. Equations. The 1-D FSI model is derived from the incompressible NavierStokes equations by making some simplifying assumptions and integrating over the cross section of the artery $\Sigma(t, z)$, where $t \in(0, T]$ is the time and $z \in[0, L]$ the axial coordinate, with $L$ the length of the vessel. The fluid equations are coupled with a structural model for the vessel wall. The vessel is assumed to be axisymmetric, and only radial displacements are considered. The pressure is assumed to be constant on each transversal section, while an axial velocity profile is imposed through a given power-law relation $[31,40]$, following the Womersley theory. The resulting state variables are

$A(t, z)=\int_{\Sigma(t, z)} \mathrm{d} \Sigma, \quad Q(t, z)=\int_{\Sigma(t, z)}\left[\boldsymbol{u}_{\mathrm{F}}\right]_{z}(t, z) \mathrm{d} \Sigma, \quad P(t, z)=\frac{1}{A(t, z)} \int_{\Sigma(t, z)} p_{\mathrm{F}}(t, z) \mathrm{d} \Sigma$,

where $A$ is the cross-sectional area, $Q$ the volumetric flow rate, $\left[\boldsymbol{u}_{\mathrm{F}}\right]_{z}$ the fluid axial velocity, and $P$ the average pressure. A derivation of the 1-D FSI model can be found 
in [39]. The resulting governing equations for continuity of mass and momentum are

$$
\left\{\begin{aligned}
\frac{\partial A}{\partial t}+\frac{\partial Q}{\partial z}=0 & \text { in }(0, L) \times(0, T], \\
\frac{\partial Q}{\partial t}+\frac{\partial}{\partial z}\left(\alpha_{\mathrm{F}} \frac{Q^{2}}{A}\right)+\frac{A}{\rho_{\mathrm{F}}} \frac{\partial P}{\partial z}+\kappa_{\mathrm{F}} \frac{Q}{A}=0 & \text { in }(0, L) \times(0, T], \\
A-\bar{A}=0, \quad Q-\bar{Q}=0 & \text { in }(0, L) \times\{0\},
\end{aligned}\right.
$$

where $\alpha_{\mathrm{F}}$ and $\kappa_{\mathrm{F}}$ are the Coriolis and friction coefficients, respectively, whose definitions are given in [31]. In (2.9) we also provide the initial values for the area $\bar{A}$ and for the volumetric flow rate $\bar{Q}$.

The fluid problem (2.9) is coupled with the 1-D structural model through the pressure-area relation, which may account for several terms [17]. In this work we consider the elastic and viscoelastic responses of the vessel wall, since the other terms provide a negligible contribution in the cardiovascular 1-D modeling context (see, e.g., $[3,31,41])$,

$$
P=\psi(A)=P_{\text {ext }}+\beta_{\mathrm{S}}\left(\sqrt{\frac{A}{A^{0}}}-1\right)+\gamma_{\mathrm{S}}\left(\frac{1}{A \sqrt{A}} \frac{\partial A}{\partial t}\right) \quad \text { in }(0, L) \times(0, T],
$$

where $P_{\text {ext }}$ is a reference external pressure (i.e., the pressure exerted by the tissues over the external wall), $A^{0}$ the reference value for the vessel area, and

$$
\beta_{\mathrm{S}}=\sqrt{\frac{\pi}{A^{0}}} \frac{h_{\mathrm{S}} E_{\mathrm{S}}}{1-\nu_{\mathrm{S}}^{2}}, \quad \gamma_{\mathrm{S}}=\frac{T_{\mathrm{S}} \tan \phi_{\mathrm{S}}}{4 \sqrt{\pi}} \frac{h_{\mathrm{S}} E_{\mathrm{S}}}{1-\nu_{\mathrm{S}}^{2}},
$$

where $h_{\mathrm{S}}$ is the wall thickness, $T_{\mathrm{S}}$ the wave characteristic time, and $\phi_{\mathrm{S}}$ the viscoelastic angle. In cardiovascular applications $T_{\mathrm{S}}$ is usually taken equal to the systolic period, while the viscoelastic angle is a parameter which provides a measure of the magnitude of the parietal viscosity force relative to the elastic one. Note that the initial condition for the average pressure can be computed by plugging $\bar{A}$ and its time derivative into (2.10). The 1-D FSI problem is finally closed by providing a proper set of boundary conditions on both sides of the model. As for the 3-D counterpart, these conditions can be either given data (for instance, expressing either a time-dependent heart flow [41] or a terminal absorbing condition [42]) or coupling conditions relating the 1-D problem with the surrounding models. We postpone the discussion of the latter case to section 3. For further details about the 1-D FSI model equations, see [31].

2.2.2. Numerical approximation. By plugging (2.10) into (2.9), we get a closed system of differential equations. On the one hand, the elastic component of the pressure-area relation is an algebraic expression and can be easily manipulated. On the other hand, the viscoelastic term depends on the temporal derivative of the area and requires a special treatment.

In the literature, several approaches have been proposed for the numerical approximation of the 1-D FSI problem, ranging from explicit discontinuous Galerkin [42] to implicit finite difference [41] or implicit finite element [3] methods. Following [17, 31, 37], in this work we use an operator splitting technique based on an explicit second order Taylor-Galerkin discretization, where the volumetric flow rate is split into two components such that $Q=\hat{Q}+\tilde{Q}$, with $\hat{Q}$ being the solution of the pure elastic problem and $\tilde{Q}$ the viscoelastic correction. On each time subinterval $\left[t^{n}, t^{n+1}\right]$, we solve the 1-D FSI problem by performing two steps. 
First step (elastic response). By removing the viscoelastic component from the formulation, we can write the closed system of equations in the classical conservative form as

$$
\frac{\partial \hat{\boldsymbol{U}}}{\partial t}+\frac{\partial \boldsymbol{F}(\boldsymbol{U})}{\partial z}+\boldsymbol{S}(\boldsymbol{U})=\mathbf{0} \quad \text { in }(0, L) \times(0, T]
$$

where $\boldsymbol{U}=[A, Q]^{\top}$ and $\hat{\boldsymbol{U}}=[A, \hat{Q}]^{\top}$ are the total and elastic conservative variables, respectively, $\boldsymbol{F}=\left[Q, F_{2}\right]^{\top}$ the corresponding fluxes, and $\boldsymbol{S}=\left[0, S_{2}\right]^{\top}$ accounts for the friction and the nonuniformity of the geometry and the material (see [31] for the detailed expression of $F_{2}$ and $S_{2}$ ). The flux $\boldsymbol{F}$ is algebraic, since it includes only the elastic part of (2.10).

Let $\boldsymbol{U}_{h}$ be the discrete counterpart of $\boldsymbol{U}$. In addition, let $\mathcal{V}_{A}$ and $\nu_{\hat{Q}}$ be two sets of piecewise linear finite element functions with adequate boundary conditions, and $\mathcal{V}_{A}^{*}$ and $\mathcal{V}_{\hat{Q}}^{*}$ be the associated linear spaces of admissible variations. From $(2.11)$ the Taylor-Galerkin formulation for the elastic problem reads: given $\boldsymbol{U}_{h}^{n}=\left[A_{h}^{n}, Q_{h}^{n}\right]^{\top}$, find $\hat{\boldsymbol{U}}_{h}^{n+1}=\left[A_{h}^{n+1}, \hat{Q}_{h}^{n+1}\right]^{\top} \in \mathcal{V}_{A} \otimes \mathcal{V}_{\hat{Q}}$ such that

$$
\begin{gathered}
\left(\hat{\boldsymbol{U}}_{h}^{n+1}, \varphi_{h}\right)=\left(\hat{\boldsymbol{U}}_{h}^{n}, \varphi_{h}\right)+\Delta t\left[\boldsymbol{F}\left(\boldsymbol{U}_{h}^{n}\right)-\frac{\Delta t}{2} \frac{\partial \boldsymbol{F}\left(\boldsymbol{U}_{h}^{n}\right)}{\partial \boldsymbol{U}}\left(\boldsymbol{S}\left(\boldsymbol{U}_{h}^{n}\right)+\frac{\partial \boldsymbol{F}\left(\boldsymbol{U}_{h}^{n}\right)}{\partial z}\right), \frac{\partial \varphi_{h}}{\partial z}\right] \\
-\Delta t\left[\boldsymbol{S}\left(\boldsymbol{U}_{h}^{n}\right)-\frac{\Delta t}{2} \frac{\partial \boldsymbol{S}\left(\boldsymbol{U}_{h}^{n}\right)}{\partial \boldsymbol{U}}\left(\boldsymbol{S}\left(\boldsymbol{U}_{h}^{n}\right)+\frac{\partial \boldsymbol{F}\left(\boldsymbol{U}_{h}^{n}\right)}{\partial z}\right), \varphi_{h}\right] \quad \forall \varphi_{h} \in \mathcal{V}_{A}^{*} \otimes \mathcal{V}_{\hat{Q}}^{*} .
\end{gathered}
$$

The discrete problem is finally closed by introducing two compatibility conditions [31].

The main advantage of this approach resides in its very low computational cost in view of the explicit nature of the numerical method (2.12). Moreover, the solution of the problem for the area (mass conservation equation) is decoupled from that for the volumetric flow rate (momentum conservation equation). However, the explicit time discretization entails a limitation on the time step related to the CFL number. In particular, the result of the von Neumann stability analysis, in the presence of a consistent mass matrix, is (see, e.g., [38])

$$
\frac{\Delta t}{h}<\frac{\sqrt{3}}{3}
$$

This is, as a matter of fact, a central issue when coupling 1-D and 3-D FSI problems, as we will see in section 3.3.2.

Second step (viscoelastic correction). By using the mass conservation equation, we remove the time dependence from the viscoelastic wall term. The resulting problem is

$$
\frac{1}{A} \frac{\partial \tilde{Q}}{\partial t}-\frac{\partial}{\partial z}\left(\frac{\gamma_{\mathrm{S}}}{\rho_{\mathrm{F}} A^{3 / 2}} \frac{\partial Q}{\partial z}\right)=0 \quad \text { in }(0, L) \times(0, T]
$$

which is closed by a proper set of homogeneous boundary conditions for $\tilde{Q}$. The corresponding finite element formulation reads: given $\left(A_{h}^{n+1}, \hat{Q}_{h}^{n+1}\right) \in \mathcal{V}_{A} \otimes \mathcal{V}_{\hat{Q}}$, find $\tilde{Q}_{h}^{n+1} \in \mathcal{V}_{\tilde{Q}}$ such that 


$$
\begin{gathered}
\left(\frac{\tilde{Q}_{h}^{n+1}}{A_{h}^{n+1}}, \varphi_{h}\right)+\Delta t\left(\frac{\gamma_{\mathrm{S}}}{\rho_{\mathrm{F}}\left(A_{h}^{n+1}\right)^{3 / 2}} \frac{\partial \tilde{Q}_{h}^{n+1}}{\partial z}, \frac{\partial \varphi_{h}}{\partial z}\right)=\left(\frac{\tilde{Q}_{h}^{n}}{A_{h}^{n+1}}, \varphi_{h}\right) \\
-\Delta t\left(\frac{\gamma_{\mathrm{S}}}{\rho_{\mathrm{F}}\left(A_{h}^{n+1}\right)^{3 / 2}} \frac{\partial \hat{Q}_{h}^{n+1}}{\partial z}, \frac{\partial \varphi_{h}}{\partial z}\right)+\Delta t\left[\frac{\gamma_{\mathrm{S}}}{\rho_{\mathrm{F}}\left(A_{h}^{n+1}\right)^{3 / 2}} \frac{\partial \hat{Q}_{h}^{n+1}}{\partial z} \varphi_{h}\right]_{0}^{L} \forall \varphi_{h} \in \mathcal{V}_{\tilde{Q}}^{*},
\end{gathered}
$$

where $\mathcal{V}_{\tilde{Q}}$ is a set of piecewise linear finite element functions with adequate boundary conditions and $\mathcal{V}_{\tilde{Q}}^{*}$ is the associated linear space of admissible variations. In (2.13) we impose either homogeneous Dirichlet or homogeneous Neumann boundary conditions, depending on the physical boundary condition types enforced at the first step. More details about the boundary conditions of the viscoelastic problem are given in [31].

3. Coupling algorithm. In this section we briefly recall from [32] the interface equations and the numerical algorithms for the partitioned solution of a geometrical multiscale problem. Then, we focus on the computation or approximation of the Jacobian entries of the interface problem. For the 3-D FSI model we devise the exact tangent problem, while for the 1-D FSI model we briefly summarize the results of [31]. The methodology presented here is devised for the coupling of the fluid part of the FSI problems. The extension to the solid part is discussed in section 4 .

3.1. Fluid interface equations. In a geometrical multiscale approach the aim is to couple several heterogeneous models without losing abstraction and generality. In particular, in section 2 we introduced two FSI models characterized by different geometrical dimensions and governed by different kinds of partial differential equations. The coupling of such different models can be set by imposing the conservation of averaged/integrated quantities over the interfaces, removing any dependency from the geometrical nature and the mathematical formulation of each model [32]. More specifically, for the fluid part of the problem these boundary quantities are the volumetric flow rate $\mathcal{Q}$ and the averaged normal component of the traction vector (mean normal stress) $\mathcal{S}$, hereafter referred to as the coupling flow and the coupling stress, respectively. On the $j$ th coupling interface of the 3-D FSI model these quantities are computed as

$$
Q_{j}^{3-\mathrm{D}}=\int_{\Gamma_{\mathrm{F}, j}^{t}} \boldsymbol{u}_{\mathrm{F}} \cdot \boldsymbol{n}_{\mathrm{F}} \mathrm{d} \Gamma, \quad \mathcal{S}_{j}^{3-\mathrm{D}}=\frac{1}{\left|\Gamma_{\mathrm{F}, j}^{t}\right|} \int_{\Gamma_{\mathrm{F}, j}^{t}}\left(\sigma_{\mathrm{F}} \cdot \boldsymbol{n}_{\mathrm{F}}\right) \cdot \boldsymbol{n}_{\mathrm{F}} \mathrm{d} \Gamma,
$$

where we assume that each coupling surface $\Gamma_{\mathrm{F}, j}^{t}, j=1, \ldots, n_{\mathrm{FS}}^{\Gamma}$, is planar.

Remark 3.1. In the present work we consider the quantity $\left(\sigma_{\mathrm{F}} \cdot \boldsymbol{n}_{\mathrm{F}}\right) \cdot \boldsymbol{n}_{\mathrm{F}}$ constant over $\Gamma_{\mathrm{F}, j}^{t}, j=1, \ldots, n_{\mathrm{FS}}^{\Gamma}$, such that we can rewrite the coupling stress as $\mathcal{S}_{j}^{3-\mathrm{D}}=$ $\left(\sigma_{\mathrm{F}} \cdot \boldsymbol{n}_{\mathrm{F}}\right) \cdot \boldsymbol{n}_{\mathrm{F}}$. Indeed, as we explain in the next remark, $\mathcal{S}_{j}^{3-\mathrm{D}}$ has the meaning of the Lagrange multiplier responsible for enforcing the imposition of the flow rate $Q_{j}^{3-D}$ through $\Gamma_{\mathrm{F}, j}^{t}, j=1, \ldots, n_{\mathrm{FS}}^{\Gamma}$.

Remark 3.2. Following the approach in [16], the imposition of the coupling flow on a coupling interface of the 3-D FSI fluid problem is addressed in a weak sense through a Lagrange multiplier. This choice leads to a uniform value of the normal component of the traction vector [25] on the same boundary interface. Moreover, it is coherent with the other chosen fluid coupling condition, i.e., the coupling stress. Note, however, that the same methodology holds when imposing the coupling flow through a given normal velocity profile. 
Remark 3.3. The 3-D FSI fluid problem needs an additional set of boundary conditions on the two tangential directions lying on $\Gamma_{\mathrm{F}, j}^{t}, j=1, \ldots, n_{\mathrm{FS}}^{\Gamma}$. Since, for modeling reasons, we assume that at these coupling interfaces the flow is fully developed and orthogonal to the plane, we impose $\left(\sigma_{\mathrm{F}} \cdot \boldsymbol{n}_{\mathrm{F}}\right) \cdot \boldsymbol{\tau}_{1 \mathrm{~F}}=0$ and $\left(\sigma_{\mathrm{F}} \cdot \boldsymbol{n}_{\mathrm{F}}\right) \cdot$ $\boldsymbol{\tau}_{2 \mathrm{~F}}=0$, where $\boldsymbol{\tau}_{1 \mathrm{~F}}$ and $\boldsymbol{\tau}_{2 \mathrm{~F}}$ are the two tangential directions.

Regarding the 1-D FSI model we have

$$
\begin{array}{ll}
\mathcal{Q}_{\mathrm{L}}^{1-\mathrm{D}}=-Q_{\mathrm{L}}, & \mathcal{S}_{\mathrm{L}}^{1-\mathrm{D}}=-P_{\mathrm{L}}, \\
\mathcal{Q}_{\mathrm{R}}^{1-\mathrm{D}}=Q_{\mathrm{R}}, & \text { on } \Gamma_{\mathrm{L}},
\end{array}
$$

where the subscripts $\mathrm{L}$ and $\mathrm{R}$ stand for left- and right-hand sides, respectively.

By using the approach described in [32], we write a set of conservation equations. More precisely, we consider a general network of models connected by $\mathcal{C}$ coupling nodes: at the $c$ th coupling node we impose the conservation of mass and the continuity of normal stresses as

$$
\left\{\begin{aligned}
\sum_{i=1}^{\mathcal{J}_{c}} \mathcal{Q}_{c, i} & =0, \\
\mathcal{S}_{c, 1}-\mathcal{S}_{c, i} & =0, \quad i=2, \ldots, \mathcal{J}_{c}
\end{aligned}\right.
$$

where $\mathcal{J}_{c}$ is the number of interfaces connected by the $c$ th coupling node, $c=1, \ldots, \mathcal{C}$.

3.2. Numerical approach. For the sake of generality, the interface problem at the $c$ th coupling node is written in a residual form. Since $\chi_{c}$ is the local vector of unknowns, the corresponding local residual form at the $c$ th coupling node is

$$
\mathcal{R}_{c}\left(\chi_{c}, \cdot\right)=\mathbf{0}
$$

with $c=1, \ldots, \mathcal{C}$, while the dot in the parentheses expresses a possible dependence of the local residual on nonlocal unknowns, i.e., quantities defined at other coupling nodes of the underlying models. The set of equations (3.1) holds independently from the type of boundary data imposed at each coupling interface, leading to an arbitrary choice of the coupling conditions to be applied on the models. Among the several possible combinations, in [32, section 2.5] three significant cases are addressed. We briefly recall two of them, the imposition of the coupling stress on all the coupling interfaces,

$$
\mathcal{R}_{c}\left(\chi_{c}, \cdot\right)=\left(\begin{array}{c}
\mathcal{Q}_{c, 1}\left(\mathcal{S}_{c}, \cdot\right)+\sum_{i=2}^{\mathcal{J}_{c}} \mathcal{Q}_{c, i} \\
\mathcal{Q}_{c, 2}\left(\mathcal{S}_{c}, \cdot\right)-\mathcal{Q}_{c, 2} \\
\mathcal{Q}_{c, 3}\left(\mathcal{S}_{c}, \cdot\right)-\mathcal{Q}_{c, 3} \\
\vdots \\
\mathcal{Q}_{c, J_{c}}\left(\mathcal{S}_{c}, \cdot\right)-\mathcal{Q}_{c, \mathcal{J}_{c}}
\end{array}\right) \quad \text { for } c \in \mathcal{L}^{\mathcal{S}}
$$


and its dual case (coupling flow imposed on all the coupling interfaces),

$$
\mathcal{R}_{c}\left(\chi_{c}, \cdot\right)=\left(\begin{array}{c}
\sum_{i=1}^{\mathcal{J}_{c}} Q_{c, i} \\
\mathcal{S}_{c, 1}\left(Q_{c, 1}, \cdot\right)-\mathcal{S}_{c, 2}\left(Q_{c, 2}, \cdot\right) \\
\mathcal{S}_{c, 1}\left(Q_{c, 1}, \cdot\right)-\mathcal{S}_{c, 3}\left(Q_{c, 3}, \cdot\right) \\
\vdots \\
\mathcal{S}_{c, 1}\left(Q_{c, 1}, \cdot\right)-\mathcal{S}_{c, J_{c}}\left(Q_{c, J_{c}}, \cdot\right)
\end{array}\right) \quad \text { for } c \in \mathcal{L}^{\mathcal{Q}}
$$

where $\mathcal{L}^{\mathcal{S}}$ and $\mathcal{L}^{\mathcal{Q}}$ denote the sets of coupling nodes where coupling stress and coupling flow conditions, respectively, are imposed on the coupling interfaces, while $\mathcal{Q}_{c, i}\left(\mathcal{S}_{c}, \cdot\right)$ and $\mathcal{S}_{c, i}\left(Q_{c, i}, \cdot\right)$ are two boundary operators that, given a proper set of coupling conditions, return the flow rate and the mean normal stress, respectively, at the $i$ th coupling interface of the $c$ th coupling node. Note that in (3.2) and (3.3) the operators are global, as they may depend also on nonlocal coupling quantities, i.e., quantities introduced at other coupling nodes. The two strategies result in the following local vectors of unknowns:

$$
\chi_{c}=\left(\mathcal{S}_{c}, Q_{c, 2}, \ldots, Q_{c, \mathcal{J}_{c}}\right)^{\top} \text { for } c \in \mathcal{L}^{\mathcal{S}}, \quad \chi_{c}=\left(Q_{c, 1}, Q_{c, 2}, \ldots, Q_{c, \mathcal{J}_{c}}\right)^{\top} \text { for } c \in \mathcal{L}^{\mathbb{Q}}
$$

Let $\chi_{\mathcal{G}}=\left(\chi_{1}^{\top}, \chi_{2}^{\top}, \ldots, \chi_{\mathcal{C}}^{\top}\right)^{\top}$ be the global vector of unknowns of all the coupling interfaces; more generally, in the following we use the subscript $\mathcal{G}$ to refer to quantities of the global interface problem. The solution of the global coupled problem is addressed by using a nonlinear Richardson strategy,

$$
\chi_{\mathcal{G}}^{k+1}=\chi_{\mathcal{G}}^{k}+\delta \chi_{\mathcal{G}}^{k}
$$

until convergence within a suitable tolerance has been achieved. The initial guess $\chi_{\mathcal{G}}^{0}$ is assigned by performing a linear extrapolation of the solution of the previous time steps. Since

$$
\boldsymbol{R}_{\mathcal{G}}\left(\chi_{\mathcal{G}}\right)=\left(\mathcal{R}_{1}\left(\chi_{1}, \cdot\right)^{\top}, \boldsymbol{R}_{2}\left(\chi_{2}, \cdot\right)^{\top}, \ldots, \mathcal{R}_{\mathcal{C}}\left(\chi_{\mathcal{C}}, \cdot\right)^{\top}\right)^{\top}
$$

are the global residuals vector of the interface problem, the update $\delta \chi_{\mathcal{G}}^{k}$ is computed by using either a Newton or an inexact-Newton method,

$$
\mathcal{J}_{\mathcal{G}}\left(\chi_{\mathcal{G}}^{k}\right) \delta \chi_{\mathcal{G}}^{k}=-\mathcal{R}_{\mathcal{G}}\left(\chi_{\mathcal{G}}^{k}\right)
$$

depending on the fact that the Jacobian matrix $\mathcal{J}_{\mathcal{G}}\left(\boldsymbol{\chi}_{\mathcal{G}}\right)$ is either exact or approximated. In both cases, the Jacobian matrix can be filled in two steps. In the first one, the Jacobian graph is built, and the required type of entries (flow rate or mean normal stress partial derivatives) are identified. This is done by using the methodology described in [32, section 3.2.1], which is devised for the most general case. Then, during the second step, the matrix entries can be either computed by solving the tangent problems associated with the coupled models [31, 32], or approximated by using a simpler finite difference approach, which may lead to a good approximation at a reasonable cost $[4,31]$. We further discuss this topic in section 3.3.

Since in the presence of 3-D FSI models the assembling of the Jacobian matrix of the interface problem may be too expensive, some further approximations can be introduced. For example, it is possible to reuse the same Jacobian matrix for 
more than one iteration and eventually more than one time step. However, a better solution in the same direction is to use a Broyden approach for the update of the Jacobian matrix at each nonlinear Richardson iteration. Indeed, this approach has been proven to perform successfully for cardiovascular applications made of networks of 1-D models [4, 31] and for heterogeneous networks of rigid fluid problems [28], requiring just a cheap evaluation of the global residuals vector of the interface problem.

3.3. Jacobian entries computation. By definition, the exact Jacobian matrix of the global interface problem is given by the derivative of the residuals vector with respect to the coupling unknowns, i.e.,

$$
\mathcal{J}_{\mathcal{G}}\left(\chi_{\mathcal{G}}\right)=\frac{\partial \mathcal{R}_{\mathcal{G}}\left(\chi_{\mathcal{G}}\right)}{\partial \chi_{\mathcal{G}}}
$$

The residuals and the unknowns vectors depend on the type of coupling conditions imposed at each node of the network. Therefore, the block structure of the Jacobian and the type of matrix entries are problem dependent. From (3.2) and (3.3) we observe that, beside the constant entries in general, there are no more than four possible coefficient types, corresponding to the partial derivatives of the two boundary operators with respect to the coupling flow and to the coupling stress, i.e.,

$$
\begin{aligned}
& \frac{\partial \mathcal{Q}_{c_{1}, i_{1}}\left(\mathcal{S}_{c_{1}}, \cdot\right)}{\partial \mathcal{Q}_{c_{2}, i_{2}}}, \quad i_{1}=1, \ldots, \mathcal{J}_{c_{1}}, \quad i_{2}=1, \ldots, \mathcal{J}_{c_{2}}, \quad \text { for } c_{1} \in \mathcal{L}^{\mathcal{S}} \text { and } c_{2} \in \mathcal{L}^{\mathcal{Q}} \\
& \frac{\partial \mathcal{Q}_{c_{1}, i_{1}}\left(\mathcal{S}_{c_{1}}, \cdot\right)}{\partial \mathcal{S}_{c_{2}}}, \quad i_{1}=1, \ldots, \mathcal{J}_{c_{1}}, \quad \text { for } c_{1} \in \mathcal{L}^{\mathcal{S}} \text { and } c_{2} \in \mathcal{L}^{\mathcal{S}} \text {, } \\
& \frac{\partial \mathcal{S}_{c_{1}, i_{1}}\left(\mathcal{Q}_{c_{1}, i_{1}}, \cdot\right)}{\partial \mathcal{Q}_{c_{2}, i_{2}}}, \quad i_{1}=1, \ldots, \mathcal{J}_{c_{1}}, \quad i_{2}=1, \ldots, \mathcal{J}_{c_{2}}, \quad \text { for } c_{1} \in \mathcal{L}^{\mathcal{Q}} \text { and } c_{2} \in \mathcal{L}^{\mathcal{Q}} \\
& \frac{\partial \mathcal{S}_{c_{1}, i_{1}}\left(\mathcal{Q}_{c_{1}, i_{1}}, \cdot\right)}{\partial \mathcal{S}_{c_{2}}}, \quad i_{1}=1, \ldots, \mathcal{J}_{c_{1}}, \quad \text { for } c_{1} \in \mathcal{L}^{\mathcal{Q}} \text { and } c_{2} \in \mathcal{L}^{\mathcal{S}} \text {, }
\end{aligned}
$$

where $c_{1}$ and $c_{2}$ are two coupling nodes inside the network. In the following, for both FSI models described in section 2 we provide a suitable numerical approach for the computation or approximation of these Jacobian entries.

3.3.1. 3-D FSI Jacobian entries. In section 2.1.3 we described a Newton method for the solution of the 3-D FSI problem with the exact computation of the FSI Jacobian matrix $\mathcal{J}_{\text {FSI }}$ in both the GCE and FI approaches. No matter which time discretization scheme is used, the Jacobian matrix of the FSI problem serves also for the computation of the Jacobian entries of the interface problems. More precisely, the discrete matrix form of the tangent problem associated with the 3-D FSI model reads

$$
\mathcal{J}_{\mathrm{FSI}}\left(\boldsymbol{u}_{\mathrm{FSI}, h}^{k}\right) \delta \boldsymbol{u}_{\mathrm{FSI}, h}=\boldsymbol{b}_{\mathrm{FSI}, j_{2}},
$$

where $\boldsymbol{u}_{\mathrm{FSI}, h}^{k}$ is the solution of the FSI problem obtained during the computation of the residuals vector of the $k$ th nonlinear Richardson iteration of the interface problem. The entries of $\boldsymbol{b}_{\mathrm{FSI}, j_{2}}$ are null apart from the contribution given by the unitary perturbation of the interface quantity applied on $\Gamma_{\mathrm{F}, j_{2}}^{t}$ such that $\delta \boldsymbol{u}_{\mathrm{FSI}, h}$ is the resulting variation of the global FSI solution vector due to that unitary perturbation. Note that this is equivalent to solving problem (2.7) with a different right-hand side that takes into account the local network topology. 
Remark 3.4. From the computational viewpoint, it is necessary to solve one tangent problem for each coupling interface of the 3-D FSI model. However, the Jacobian matrix is the same for all the cases. Moreover, it is already available from the previous Newton iterations of the FSI problem (see (2.6)), where it has been computed by using the second-to-last state variables, i.e., the state variables at the iteration before the convergence.

Remark 3.5. In the GCE time discretization approach, problem (2.8) is solved just once per time step, at the first nonlinear Richardson iteration of the interface problem. Indeed, it does not depend on any fluid coupling quantity.

The values of the Jacobian entries are then retrieved from the solution of the FSI tangent problem. More precisely, they are computed from $\delta \boldsymbol{u}_{\mathrm{FSI}, h}$ as the resulting flow rate and mean normal stress on $\Gamma_{\mathrm{F}, j_{1}}^{t}$.

3.3.2. 1-D FSI Jacobian entries. In section 2.2 .2 we briefly discussed the limitation imposed on the time step by the CFL condition. This limitation is particularly severe when coupling the 1-D FSI model with computationally expensive 3-D models, e.g., the 3-D FSI model described in section 2.1. In fact, to perform the nonlinear Richardson iterations on the global coupled problem, all the elements in the network must use the same time step; thus the element that requires the smallest time step forces the entire system to advance the computations at its own time step. This goes in the opposite direction with respect to the need for a large time step for the coupled 3 -D FSI problem, in order to minimize the computational cost. For instance, when solving the blood flow in an arterial network, the time step required for the solution of the 1-D FSI segments in the circle of Willis may be around two orders of magnitude smaller than the one needed by a 3-D FSI vessel embedded in the arterial tree.

This problem is addressed in [31] by introducing a two-level time step technique. More precisely, the inner time step meets the local 1-D CFL requirements, being different for each 1-D FSI model, while the outer time step is used for the global coupling between the models; i.e., (3.1) is satisfied just at this outer level. At the inner time level, the boundary conditions for the 1-D problems are provided by using a Lagrange polynomial interpolation of the coupling conditions. This approach allows us to choose the outer global time step according to the requirement of any 3-D FSI problem in the network.

The computation of the Jacobian coefficients of the 1-D FSI problem can be addressed by solving the associated exact tangent problem, as described in [31]. However, in the presence of inner time steps, the recursion of the problem creates an obstacle that prevents reaching an analytical expression of these entries. A possible alternative is to devise an approximated version of the tangent problem. Nevertheless, as shown in [31], a finite difference approach is more effective in this case. By following this latter strategy, the derivative of the mean normal stress on the left-hand side of the 1-D model with respect to the coupling flow on the same side is given by

$$
\frac{\partial \mathcal{S}_{\mathrm{L}}\left(\mathrm{Q}_{\mathrm{L}}, \cdot\right)}{\partial \mathcal{Q}_{\mathrm{L}}} \cong \frac{\mathcal{S}_{\mathrm{L}}\left(\mathrm{Q}_{\mathrm{L}}^{n+1}+\delta \mathrm{Q}_{\mathrm{L}}, \cdot\right)-\mathcal{S}_{\mathrm{L}}\left(\mathrm{Q}_{\mathrm{L}}^{n+1}, \cdot\right)}{\delta \mathrm{Q}_{\mathrm{L}}},
$$

where $\delta \mathrm{Q}_{\mathrm{L}}$ is a properly chosen volumetric flow rate perturbation parameter and the dot represents the nonlocal coupling condition, i.e., the coupling condition on the right-hand side of the 1-D segment. The other entry types are addressed similarly. For more details, see [31].

4. Interface conditions for the 3-D solid problem. As discussed in section 2.1, problem (2.3) requires $n_{\mathrm{FS}}^{\Gamma}$ boundary conditions on the inlet and outlet solid 
rings $\Gamma_{\mathrm{S}, j}^{0}, j=1, \ldots, n_{\mathrm{FS}}^{\Gamma}$. These boundary conditions can be either provided from data, or computed by using information related to the surrounding models. In this section, we propose three different approaches to closing the solid problem. For each approach we highlight the main pros and cons. The analysis of the numerically induced wave reflections in a geometrical multiscale setting is deferred until section 5 .

4.1. Fixed solid ring boundary condition. A first approach, widely used in the literature, consists of fixing the solid rings of the 3-D FSI model by imposing a homogeneous Dirichlet boundary condition on the solid displacement, i.e.,

$$
\boldsymbol{d}_{\mathrm{S}}=\mathbf{0} \quad \text { on } \Gamma_{\mathrm{S}, j}^{0} \times(0, T]
$$

with $j=1, \ldots, n_{\mathrm{FS}}^{\Gamma}$. This approach is very easy to implement, since no additional equations are required. However, this type of boundary condition is far from representing the behavior of the arterial wall at those locations. First of all, the value of the solid displacement near the fixed rings cannot be considered reliable; neither are the values of other physical quantities such as the wall stress state. In addition, spurious backward reflections may be generated at the outlets of the 3-D domain; in a geometrical multiscale setting, this problem can also affect the surrounding 1-D FSI models.

4.2. Free solid ring boundary condition. More realistic behavior of the arterial wall at the inlet/outlet rings is in principle obtained by imposing homogeneous Neumann boundary conditions on the normal component of the first Piola-Kirchhoff stress tensor, i.e.,

$$
\sigma_{\mathrm{S}} \cdot \boldsymbol{n}_{\mathrm{S}}=\mathbf{0} \quad \text { on } \Gamma_{\mathrm{S}, j}^{0} \times(0, T]
$$

with $j=1, \ldots, n_{\mathrm{FS}}^{\Gamma}$. However, this approach may lead to an ill-posed problem, since the six rigid-body modes of the vessel are not all constrained. In addition, from the physical viewpoint, the continuation of the vessel beyond the boundaries is not represented; in particular, the solid rings are free to move along the longitudinal direction. To address these issues, we devise a different approach, i.e.,

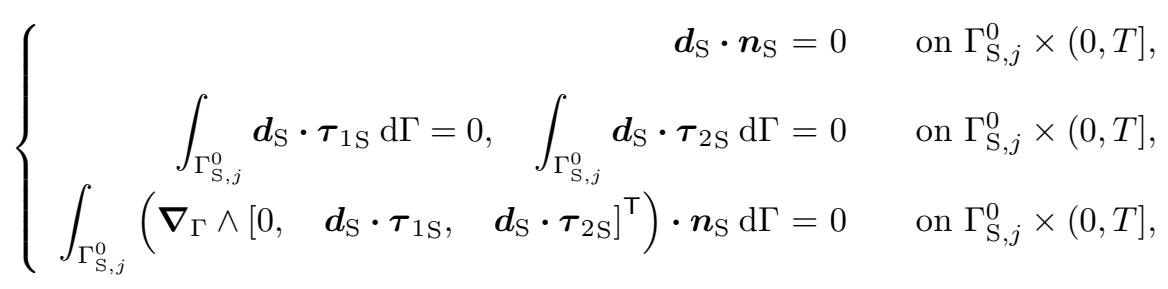

where $\boldsymbol{\tau}_{1 \mathrm{~S}}$ and $\boldsymbol{\tau}_{2 \mathrm{~S}}$ are the two tangential directions lying on $\Gamma_{\mathrm{S}, j}^{0}, j=1, \ldots, n_{\mathrm{FS}}^{\Gamma}$. The first restriction prevents the normal displacement of the solid ring (in the longitudinal direction), representing the continuation of the vessel beyond the domain boundaries. In addition, it removes three rigid-body modes: the normal translation and the two nonplanar rotations. The second restriction removes the two planar translation rigidbody modes without imposing any constraint on the area of the vessel. More precisely, the boundary rings can enlarge or reduce their size around a fixed geometric center. This restriction can be rewritten in the following form:

$$
\int_{\Gamma_{\mathrm{S}, j}^{0}} \mathrm{P} \boldsymbol{d}_{\mathrm{S}} \mathrm{d} \Gamma=\mathbf{0} \quad \text { on } \Gamma_{\mathrm{S}, j}^{0} \times(0, T]
$$


with $\mathrm{P}=\mathrm{I}-\boldsymbol{n}_{\mathrm{S}} \cdot \boldsymbol{n}_{\mathrm{S}}^{\top}$ the local projection operator over the tangential plane where each solid ring lies. Finally, the third restriction removes the planar rotation rigid-body mode. Indeed, it can also be rewritten as

$$
\int_{\Gamma_{\mathrm{S}, j}^{0}}\left(\nabla_{\Gamma} \wedge \mathrm{P} \boldsymbol{d}_{\mathrm{S}}\right) \cdot \boldsymbol{n}_{\mathrm{S}} \mathrm{d} \Gamma=0 \quad \text { on } \Gamma_{\mathrm{S}, j}^{0} \times(0, T]
$$

Note that $\mathrm{P} \boldsymbol{d}_{\mathrm{S}}$ is a vector lying on the two-dimensional plane defined by its normal $\boldsymbol{n}_{\mathrm{S}}$, while $\nabla_{\Gamma} \wedge$ denotes the curl operator defined in terms of the tangential coordinates and applied to such a tangent vector, whose action yields a vector aligned with $\boldsymbol{n}_{\mathrm{S}}$. Furthermore, we can use a variant of the Green theorem to change the last restriction into the following:

$$
\int_{C_{\mathrm{S}, j}^{0}} \mathrm{P} \boldsymbol{d}_{\mathrm{S}} \cdot \boldsymbol{\tau}_{C} \mathrm{~d} C=0 \quad \text { on } C_{\mathrm{S}, j}^{0} \times(0, T]
$$

where $C_{\mathrm{S}, j}^{0}$ denotes the internal and external curves that define the ring $\Gamma_{\mathrm{S}, j}^{0}, j=$ $1, \ldots, n_{\mathrm{FS}}^{\Gamma}$, and $\boldsymbol{\tau}_{C}$ is the tangent vector to these curves.

Remark 4.1. In [9] evidence of a significant longitudinal component of the motion of the arterial wall in some vessels (e.g., the carotid artery) was provided. In contrast to the current conjecture, they observed a distinct longitudinal movement of the arterial wall which is of the same magnitude as the local diameter change for a healthy patient. In view of this result, the first restriction in (4.3) can be either modified to prescribe a given longitudinal displacement field obtained from, e.g., a lumped parameters model, or relaxed through a Robin boundary condition.

Remark 4.2. In the most general case, where there exists at least one solid ring whose normal is not aligned with one of the Cartesian axes, it is convenient to impose the three restrictions by introducing different Lagrange multipliers in the weak form of the solid problem.

Remark 4.3. If there exist at least three boundary solid rings equipped with a different normal (as, e.g., in the case of a bifurcating artery), all the six rigid-body modes can be removed by imposing just the first restriction in (4.3). However, from the physical point of view, the other two restrictions are still relevant.

The main advantage of this approach compared to (4.1) is the very general way of addressing the boundary conditions for the 3-D solid problem, without overconstraining the structure displacement at the coupling interfaces. In particular, it suits both stand-alone 3-D FSI problems and geometrical multiscale strategies, where the 3-D domain is coupled with 1-D problems although the continuity of the area is not guaranteed.

4.3. Scaled area solid ring boundary condition. A third different strategy is to prescribe the value of the boundary area of each fluid coupling interface. Following the approach devised in [19], this can be done by prescribing a radial displacement of the internal contour of the $j$ th 3 -D solid ring, $j=1, \ldots, n_{\mathrm{FS}}^{\Gamma}$, i.e.,

$$
\left\{\begin{aligned}
\boldsymbol{d}_{\mathrm{S}} \cdot \boldsymbol{n}_{\mathrm{S}}=0 & \text { on } \Gamma_{\mathrm{I}}^{0} \cap \Gamma_{\mathrm{S}, j}^{0} \times(0, T], \\
{\left[\boldsymbol{d}_{\mathrm{S}}-\Psi_{j}^{t}\left(\boldsymbol{x}^{0}-\boldsymbol{x}_{\mathrm{G}, j}^{0}\right)\right] \cdot \boldsymbol{\tau}_{1 \mathrm{~S}}=0 } & \text { on } \Gamma_{\mathrm{I}}^{0} \cap \Gamma_{\mathrm{S}, j}^{0} \times(0, T], \\
{\left[\boldsymbol{d}_{\mathrm{S}}-\Psi_{j}^{t}\left(\boldsymbol{x}^{0}-\boldsymbol{x}_{\mathrm{G}, j}^{0}\right)\right] \cdot \boldsymbol{\tau}_{2 \mathrm{~S}}=0 } & \text { on } \Gamma_{\mathrm{I}}^{0} \cap \Gamma_{\mathrm{S}, j}^{0} \times(0, T],
\end{aligned}\right.
$$


which corresponds to a geometric rescaling of the surface, where the radial scale factor is defined as

$$
\Psi_{j}^{t}=\sqrt{\frac{A_{j}^{t}}{A_{j}^{0}}}-1,
$$

with $A_{j}^{0}$ and $\boldsymbol{x}_{\mathrm{G}, j}^{0}$ being the reference area of the $j$ th coupling interface of the 3-D fluid problem and its geometric center, respectively. This approach preserves the original shape of each 3-D solid ring, whose size is scaled by the value of the given boundary area $A_{j}^{t}$. Note that in (4.4) the normal and tangent vectors are defined on the $j$ th solid ring $\Gamma_{\mathrm{S}, j}^{0}$.

Remark 4.4. As (4.3) does, (4.4) constrains all the rigid-body modes of the 3-D FSI problem. However, this condition is applied only to the inner contour of the boundary rings, i.e., $\Gamma_{\mathrm{I}}^{0} \cap \Gamma_{\mathrm{S}, j}^{0}, j=1, \ldots, n_{\mathrm{FS}}^{\Gamma}$. To close the 3 -D FSI solid problem, we need to impose an additional boundary condition on $\Gamma_{\mathrm{S}, j}^{0} \backslash \Gamma_{\mathrm{I}}^{0} \cap \Gamma_{\mathrm{S}, j}^{0} \times(0, T], j=$ $1, \ldots, n_{\mathrm{FS}}^{\Gamma}$, which can be either (4.2) or (4.3). The former leaves some degrees of freedom to the displacement of the boundary solid rings; e.g., the external contour of the rings can move in the longitudinal direction or rotate on itself. However, if the thickness of the solid wall is small with respect to the vessel area, these movements are negligible.

Since, in general, boundary data of the vessel area are not available for cardiovascular simulations, the value of the area can be obtained either by modeling its dynamic through a local simple model, or, in a geometrical multiscale setting, by imposing the continuity of the boundary area with the surrounding models. The latter case is explored in the next section. Regarding the former, the dynamic of the local boundary area can be expressed as a function of the time and of some local boundary quantities, e.g., volumetric flow rate and average pressure. Among the several possibilities, a straightforward choice is to use the inverse of the 1-D pressure-area relation described in (2.10), eventually adding some further contributions, such as the inertia of the wall, in order to match the physics of the 3-D solid problem given in (2.3).

4.3.1. Continuity of the vessel area. In a geometrical multiscale setting, the interfaces of the 3-D FSI model are generally connected with those of reduced models, such as the 1-D FSI model. In this case, it is possible to write an additional relation at the coupling interfaces of the two heterogeneous FSI models to impose the continuity of the area of the connected vessels. To do this, let us first introduce a new averaged quantity on each boundary interface, i.e, the area of the fluid section $\mathcal{A}$, hereafter referred to as the coupling area. On the $j$ th coupling interface of the 3-D FSI this quantity is computed as

$$
\mathcal{A}_{j}^{3-\mathrm{D}}=\left|\Gamma_{\mathrm{F}, j}^{t}\right|,
$$

with $j=1, \ldots, n_{\mathrm{FS}}^{\Gamma}$. Regarding the 1-D FSI model it is given by

$$
\mathcal{A}_{\mathrm{L}}^{1-\mathrm{D}}=A_{\mathrm{L}}, \text { on } \Gamma_{\mathrm{L}}, \quad \mathcal{A}_{\mathrm{R}}^{1-\mathrm{D}}=A_{\mathrm{R}}, \text { on } \Gamma_{\mathrm{R}} .
$$

Let $\mathcal{L}^{\mathcal{A}}$ be the set of coupling nodes where the continuity of the area between the two heterogeneous FSI models is imposed. There, (3.1) becomes

$$
\left\{\begin{array}{l}
\mathcal{Q}_{c, 1}^{1-\mathrm{D}}+\mathcal{Q}_{c, 2}^{3-\mathrm{D}}=0 \\
\mathcal{S}_{c, 1}^{1-\mathrm{D}}-\mathcal{S}_{c, 2}^{3-\mathrm{D}}=0 \\
\mathcal{A}_{c, 1}^{1-\mathrm{D}}-\mathcal{A}_{c, 2}^{3-\mathrm{D}}=0
\end{array}\right.
$$




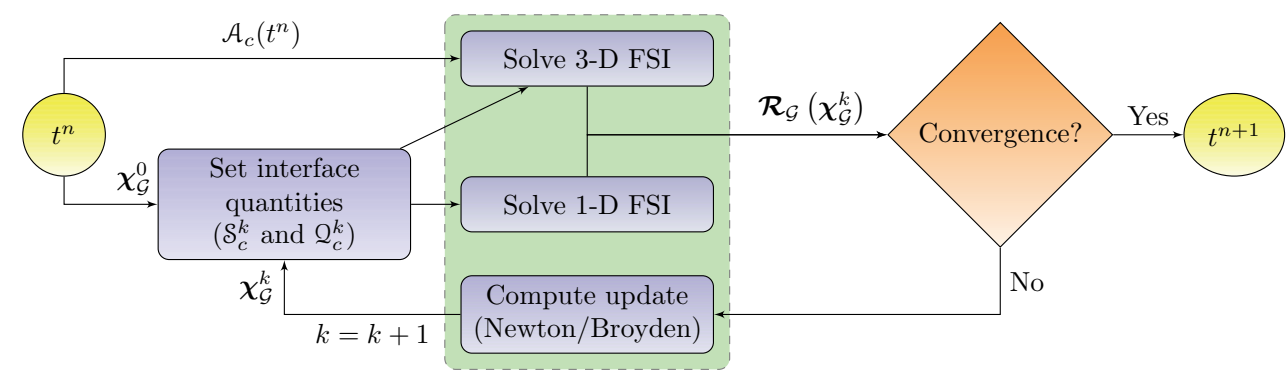

FIG. 4.1. Algorithm flow diagram for the coupling of two geometrically heterogeneous FSI models, using the explicit time discretization scheme for the continuity of the vessel area. In the diagram $\chi_{\mathcal{G}}$ includes only fluid quantities, i.e., $\chi_{c}=\left(\mathcal{S}_{c}, Q_{c, 2}\right)^{\top}$ for $c \in \mathcal{L}^{\mathcal{S}} \cap \mathcal{L}^{\mathcal{A}}$ and $\chi_{c}=\left(Q_{c, 1}, Q_{c, 2}\right)^{\top}$ for $c \in \mathcal{L}^{\mathcal{Q}} \cap \mathcal{L}^{\mathcal{A}}$.

where $c \in \mathcal{L}^{\mathcal{A}}$ and, for the sake of clarity, the model to which each quantity belongs is indicated in the superscript. We remark that (4.6) is written for the specific case of a 3 -D FSI interface coupled with a single 1-D FSI model. In the case of a generalization to two or more 1-D models connected to the same 3-D FSI interface setting, the continuity of the area does not make sense, and for this reason we do not address this case. Indeed, one of the two sets of interface equations proposed in section 3.2 should be employed in these situations, together with one of the proposed solid ring boundary conditions (i.e., (4.1), (4.3), and (4.4)) applied to the corresponding 3-D FSI interface. However, to avoid this problem, we suggest treating bifurcations or similar topologies as either single 3-D geometries or networks of 1-D segments.

As described in section 2.2, the 1-D FSI problem needs just one physical boundary condition on each side of the segment; by imposing either the coupling flow or the coupling stress, the value of the coupling area of the 1-D segment is already determined by the solution of the 1-D problem. From the numerical viewpoint, this creates the opportunity to use three different time discretization schemes to solve the problem at the coupling interfaces.

Explicit. In cardiovascular simulations the area of the vessel at the boundaries changes very smoothly in time. We can therefore treat it explicitly, in which case the area of each interface of the 3-D FSI model at time $t^{n+1}$ is imposed equal to that of the nearby 1-D interface at the previous time step. By using this approach, each radial scale factor $\Psi_{j}^{t}, j=1, \ldots, n_{\mathrm{FS}}^{\Gamma}$, is computed explicitly at the beginning of each time step. Therefore, the residual form at the coupling interfaces is still written just in terms of flow rate and mean normal stress, as in (3.2) and (3.3). The flow diagram of this scheme is shown in Figure 4.1.

Implicit with hierarchy. A different strategy for preserving the implicit coupling of the area of the vessel without adding an equation in the residual form is to introduce a hierarchy among the models such that at each nonlinear Richardson iteration first we solve the 1-D FSI models by computing the boundary area of each 1-D segment, and then we use these values to close and solve the nearby 3-D FSI models. As in the explicit case, the residual form at the coupling interfaces is written in terms of the coupling flow and the coupling stress or the coupling total stress. However, the radial scale factor $\Psi_{j}^{t}, j=1, \ldots, n_{\mathrm{FS}}^{\Gamma}$, is updated with the new value of the area of the 1-D problem at each iteration of the interface problem. Since the dependence on the varying area is not taken into account in the 3 -D boundary operators, the resulting 


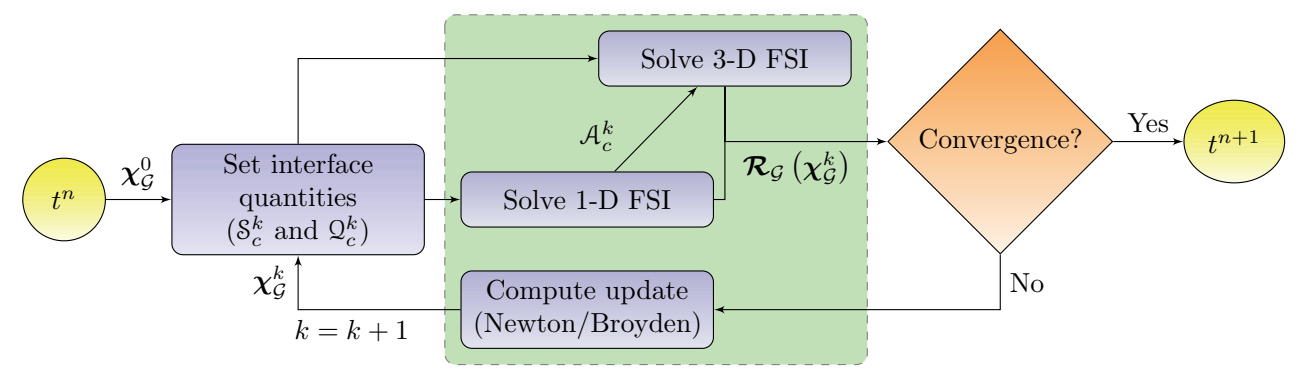

FIG. 4.2. Algorithm flow diagram for the coupling of two geometrically heterogeneous FSI models, using the implicit-with-hierarchy time discretization scheme for the continuity of the vessel area. In the diagram $\boldsymbol{\chi}_{\mathcal{G}}$ includes only fluid quantities, i.e., $\boldsymbol{\chi}_{c}=\left(\mathcal{S}_{c}, Q_{c, 2}\right)^{\top}$ for $c \in \mathcal{L}^{\mathcal{S}} \cap \mathcal{L}^{\mathcal{A}}$ and $\chi_{c}=\left(Q_{c, 1}, Q_{c, 2}\right)^{\top}$ for $c \in \mathcal{L}^{\mathcal{Q}} \cap \mathcal{L}^{\mathcal{A}}$.

Jacobian matrix is inexact and might lead to an increase in the number of iterations. Another drawback is that, due to the introduced hierarchy, the models cannot be solved at the same time on different machines. Nevertheless, if convergence is achieved, both this and the original nonsimplified approaches yield the same solution. The flow diagram of this scheme is shown in Figure 4.2.

Implicit without hierarchy. As in the previous case, the area of each interface of the 3-D FSI model at time $t^{n+1}$ is imposed equal to that of the nearby 1-D interface at the same time step. However, instead of introducing a hierarchy among the models, we impose the continuity of the area in the residual formulation such that (3.2) becomes

$$
\mathcal{R}_{c}\left(\chi_{c}, \cdot\right)=\left(\begin{array}{r}
\mathcal{Q}_{c, 1}^{1-D}\left(\mathcal{S}_{c}, \cdot\right)+Q_{c} \\
\mathcal{Q}_{c, 2}^{3-D}\left(\mathcal{S}_{c}, \mathcal{A}_{c}, \cdot\right)-\mathcal{Q}_{c} \\
\mathcal{A}_{c, 1}^{1-D}\left(\mathcal{S}_{c}, \cdot\right)-\mathcal{A}_{c}
\end{array}\right) \quad \text { for } c \in \mathcal{L}^{\mathcal{S}} \cap \mathcal{L}^{\mathcal{A}}
$$

and (3.3) becomes

$$
\mathcal{R}_{c}\left(\boldsymbol{\chi}_{c}, \cdot\right)=\left(\begin{array}{c}
Q_{c, 1}+Q_{c, 2} \\
\mathcal{S}_{c, 1}^{1-D}\left(Q_{c, 1}, \cdot\right)-\mathcal{S}_{c, 2}^{3-D}\left(Q_{c, 2}, \mathcal{A}_{c}, \cdot\right) \\
\mathcal{A}_{c, 1}^{1-D}\left(Q_{c, 1}, \cdot\right)-\mathcal{A}_{c}
\end{array}\right) \quad \text { for } c \in \mathcal{L}^{\mathcal{Q}} \cap \mathcal{L}^{\mathcal{A}},
$$

where $\mathcal{A}_{c, i}(\cdot, \cdot)$ is a boundary operator that, given a proper set of coupling conditions, returns the coupling area at the $i$ th coupling interface of the $c$ th coupling node.

Remark 4.5. In the development discussed in section 3 the conservation equations do not imply any specific choice of the coupling conditions, leaving the flexibility to impose either the coupling flow or the coupling stress on each coupling interface. In contrast, the set of equations (4.6) requires particular care due to the presence of the coupling area. More precisely, the coupling area can be imposed only on the 3-D FSI problem, since the 1-D FSI model needs just one physical boundary condition on each side of the segment. Therefore, the third row in (4.7) and (4.8) cannot be replaced with an equivalent version written by replacing the 1-D boundary area operator with the 3-D one. For instance, in terms of components, in (4.7) notice that, at the $c$ th interface, the 3 -D model receives as input $\left(\mathcal{S}_{c}, \mathcal{A}_{c}\right)$ and yields as output $\mathcal{Q}_{c, 2}^{3-\mathrm{D}}$, while the 1-D model receives $\mathcal{S}_{c}$ and gives back $\left(\mathcal{Q}_{c, 1}^{1-\mathrm{D}}, \mathcal{A}_{c, 1}^{1-\mathrm{D}}\right)$.

The two implicit coupling strategies result in the following local vectors of unknowns:

$$
\chi_{c}=\left(\mathcal{S}_{c}, Q_{c}, \mathcal{A}_{c}\right)^{\top} \text { for } c \in \mathcal{L}^{\mathcal{S}} \cap \mathcal{L}^{\mathcal{A}}, \quad \chi_{c}=\left(Q_{c, 1}, Q_{c, 2}, \mathcal{A}_{c}\right)^{\top} \text { for } c \in \mathcal{L}^{\mathbb{Q}} \cap \mathcal{L}^{\mathcal{A}}
$$




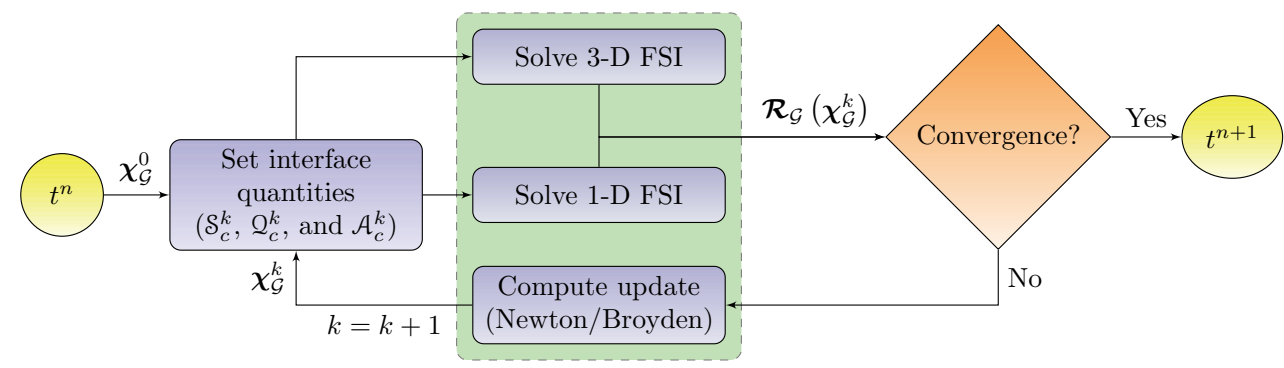

FIG. 4.3. Algorithm flow diagram for the coupling of two geometrically heterogeneous FSI models, using the implicit-without-hierarchy time discretization scheme for the continuity of the vessel area. In the diagram, $\boldsymbol{\chi}_{\mathcal{G}}$ includes both the fluid quantities and the vessel area, i.e., $\boldsymbol{\chi}_{c}=$ $\left(\mathcal{S}_{c}, Q_{c, 2}, \mathcal{A}_{c}\right)^{\top}$ for $c \in \mathcal{L}^{\mathcal{S}} \cap \mathcal{L}^{\mathcal{A}}$ and $\boldsymbol{\chi}_{c}=\left(Q_{c, 1}, Q_{c, 2}, \mathcal{A}_{c}\right)^{\top}$ for $c \in \mathcal{L}^{\mathcal{Q}} \cap \mathcal{L}^{\mathcal{A}}$.

such that the solution of the global interface problem can again be addressed by using a nonlinear Richardson approach, as in (3.4). With respect to the development presented in section 3.3, the only difference consists of the dependence of the residual on the coupling area, such that four new Jacobian entry types need to be computed/approximated to fill the Jacobian matrix in the most general case:

- $\frac{\partial \mathcal{Q}_{c_{1}}\left(\mathcal{S}_{c_{1}}, \mathcal{A}_{c_{1}}, \cdot\right)}{\partial \mathcal{A}_{c_{2}}}$ and $\frac{\partial \mathcal{S}_{c_{1}}\left(Q_{c_{1}}, \mathcal{A}_{c_{1}}, \cdot\right)}{\partial \mathcal{A}_{c_{2}}}$ belong just to the 3-D FSI model and can be computed by solving the associated tangent problem;

- $\frac{\partial \mathcal{A}_{c_{1}}\left(\mathcal{S}_{c_{1}}, \cdot\right)}{\partial \mathcal{S}_{c_{2}}}$ and $\frac{\partial \mathcal{A}_{c_{1}}\left(Q_{c_{1}}, \cdot\right)}{\partial Q_{c_{2}}}$ belong just to the 1-D FSI model and can be approximated by using a finite difference technique.

Remark 4.6. The assembling procedure of the Jacobian matrix and the detailed description of the diagonal and off-diagonal blocks for the nodes where $c \in \mathcal{L}^{\mathcal{A}}$ are extensively described in [30, Chapter 3] and not repeated here.

The flow diagram of this scheme is shown in Figure 4.3. One of the main advantages of this approach over the previous one is that no hierarchy must be established among the models such that, in a parallel framework, each model can be solved at the same time on different machines. In addition, the Jacobian matrix of the interface problem is still exact, except for other approximations introduced at the numerical level, i.e., the finite difference approximation of the 1-D FSI entries.

Remark 4.7. If convergence is achieved, both the implicit-with-hierarchy and the implicit-without-hierarchy approaches yield the same solution.

4.4. Qualitative comparison of the solid boundary conditions. Finally, it is worthwhile to summarize the main pros and cons of the three methodologies described above. This comparison is shown in Table 4.1. For the case of a geometrical multiscale problem, a quantitative analysis of the wave reflections at the coupling interfaces is addressed in section 5 .

5. Wave reflection analysis in a series of heterogeneous FSI pipes. In this section, we make use of simple benchmark examples to compare the behavior of the three proposed interface conditions introduced in section 4 for the closure of the 3-D solid problem. In particular, we focus our analysis on the spurious backward wave reflections that might be generated at the coupling interfaces between two dimensionally heterogeneous pipes. In order to have a full overview of the phenomena we analyze both the case of a 1-D wave flow that enters in a $3-\mathrm{D}$ pipe and the inverse, i.e., a 3-D wave flow that enters in a 1-D pipe. In both cases, on the leftmost inflow 
TABLE 4.1

Qualitative comparison of the main pros and cons of each proposed interface ring boundary condition for the 3-D solid problem. Note that in the third column some comments depend on the chosen approach (local model versus continuity of the vessel area).

\begin{tabular}{lccc}
\hline & Fixed & Free & Scaled area \\
\hline Software implementation complexity & Very low & High & Medium \\
Physics at the boundaries & Unrealistic & Realistic & Realistic \\
Continuity of the vessel area & No & No & No/Yes \\
Usable in stand-alone 3-D FSI problems & Yes & Yes & Yes/No \\
Usable in geometrical multiscale problems & Yes & Yes & Yes \\
\hline
\end{tabular}

we impose a single flow rate wave defined as

$$
Q(t)= \begin{cases}-\sin \left(\frac{2 \pi t}{T_{\mathrm{w}}}\right)^{2}, & t<\frac{T_{\mathrm{w}}}{2}, \\ 0 & \text { otherwise }\end{cases}
$$

where $T_{\mathrm{w}}=0.005$ is the chosen wave period. On the rightmost outflow, a proper absorbing boundary condition is applied [35, 42]. The interface problem is defined by imposing the coupling stress on the coupling interfaces. In the following, unless otherwise specified, the reference dimensionless parameters that define the problem are $\rho_{\mathrm{F}}=1, \mu_{\mathrm{F}}=0.035, \alpha_{\mathrm{F}}=1.1, A^{0}=\pi, h_{\mathrm{S}}=0.1, E_{\mathrm{S}}=3000000, \rho_{\mathrm{S}}=0$, and $\gamma_{\mathrm{S}}=0$. Note that with this choice of $\rho_{\mathrm{S}}$ and $\gamma_{\mathrm{S}}$, the inertia and the viscoelastic effects of the wall are neglected in both FSI models; indeed, we aim to study the reflections generated by the dimensional heterogeneity of the models, and thus we need to exclude other possible sources of reflections, such as heterogeneous physical modeling features. The Poisson ratio $\nu_{\mathrm{S}}$ is 0.5 for the 1-D FSI model and 0.475 for the 3 -D FSI model. The length $L$ of each pipe is equal to 3 . On the solid external wall $\Gamma_{\mathrm{S}, \text { ext }}^{0}$ of the 3-D FSI problem we apply a homogeneous Neumann boundary condition, while we set $P_{\text {ext }}=0$ in the 1-D FSI problem.

The imposed tolerance for the interface problem is $10^{-6}$, while that for the 3-D FSI problem is $10^{-7}$. The mesh of the fluid part of the 3-D pipe consists of 137712 tetrahedral elements with 25077 vertices, while the solid part is made of 51984 tetrahedral elements with 11856 vertices. The corresponding average space discretization for the fluid and solid problems is 0.094 and 0.092 , respectively. Regarding the 1-D FSI pipe, we use a uniform space discretization of 0.1 , corresponding to 31 vertices. For both models the global time step is set equal to $10^{-5}$; since this is quite a small value, we use the GCE time discretization scheme, introduced in section 2.1.3, to solve the 3-D FSI problem. All the simulations last 1500 time steps $(T=0.0015)$, which is the time needed by the flow wave to reach the rightmost side of the problems.

5.1. From 1-D to $3-D$ wave flow propagation. The first case we analyze is described in Figure 5.1(a): a wave propagates from a 1-D FSI segment (on the left) to a 3-D FSI pipe (on the right). As a reference case we consider the solution computed by solving the problem with a single 1-D segment of length 6 (see Figure 5.1(b)). By comparing the solution of the two problems we study the reflection amplitude generated at the coupling interfaces due to the heterogeneity of the models.

The results are summarized in Figure 5.2. On the $x$-axis we represent the normalized time, while on the $y$-axis we plot the difference between the volumetric flow rate in the heterogeneous case (see Figure 5.1(a)) and the rate given by the reference 


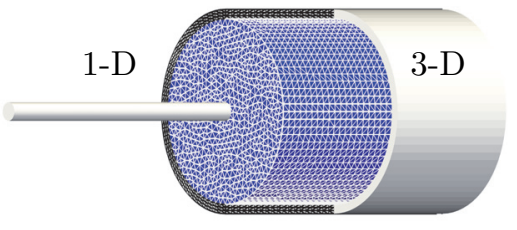

(a)
$1-\mathrm{D}$

(b)

FIG. 5.1. Schematic representation of the benchmark case. (a) The wave flow propagates from the 1-D FSI model to the 3-D FSI one. The length of each pipe is equal to 3. (b) The reference case: A single 1-D FSI pipe of length 6.

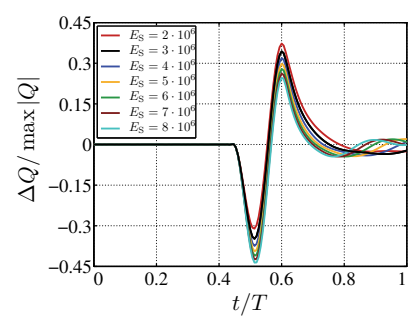

(a)

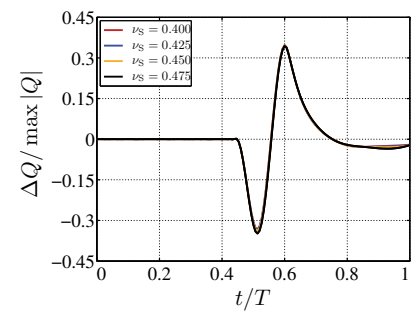

(d)

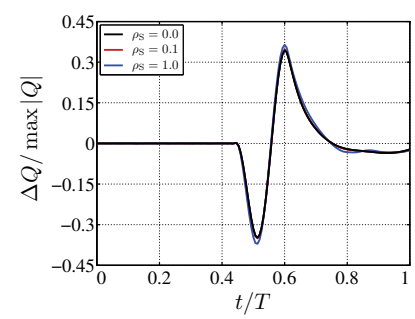

(g)

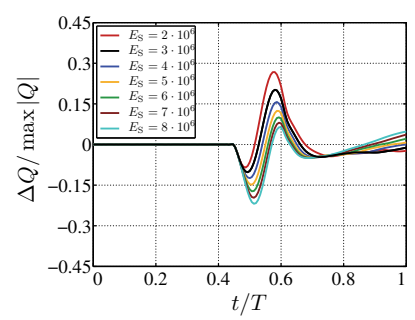

(b)

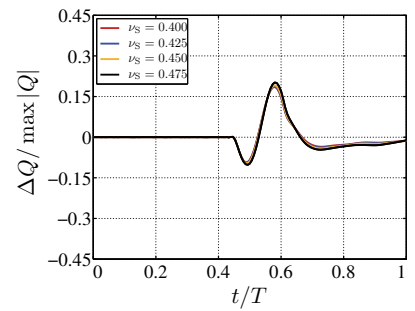

(e)

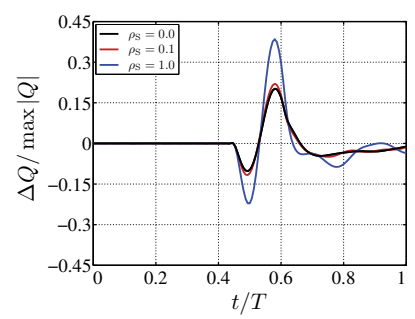

(h)

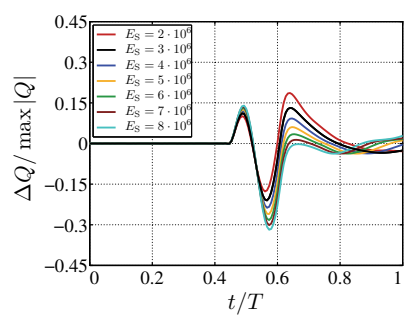

(c)

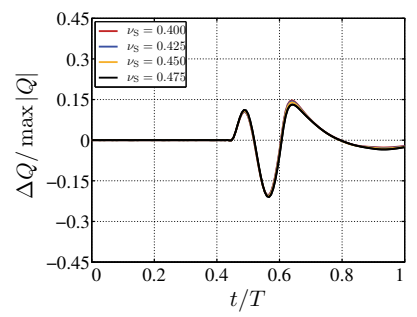

(f)

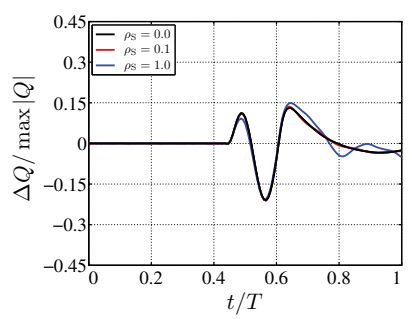

(i)

FIG. 5.2. Volumetric flow rate wave reflection at the coupling interfaces for different values of the main 3-D solid parameters. In all the figures, the black curve corresponds to the reference parameters. (a,b,c) Varying Young's modulus. (d,e,f) Varying Poisson's ratio. (g,h,i) Varying solid density. (a,d,g) Fixed area boundary condition on the $3-D$ solid ring. (b,e,h) Free area boundary condition on the 3-D solid ring. (c,f,i) Continuity of the area at the coupling interfaces.

case (see Figure 5.1(b)), namely $\Delta Q$, normalized over the maximum amplitude of the inflow wave. This quantity coincides with the normalized reflection wave generated at the coupling interface. Note that a similar analysis done in terms of pressure waves (which for brevity is not presented here) leads to the same conclusions.

First we observe that, as expected, the amplitude of the reflection is always larger 


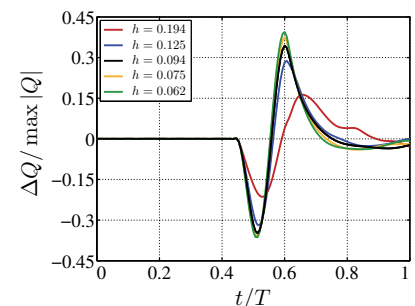

(a)

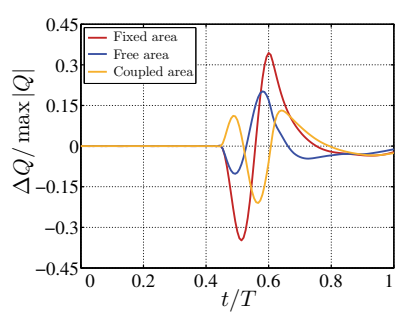

(d)

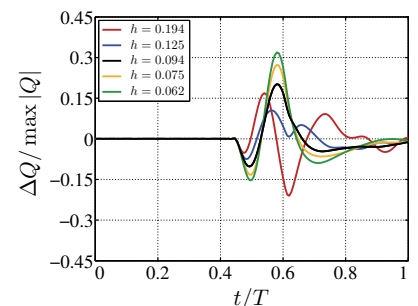

(b)

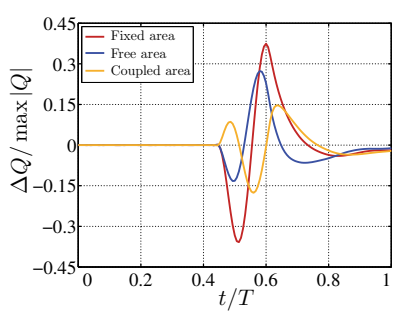

(e)

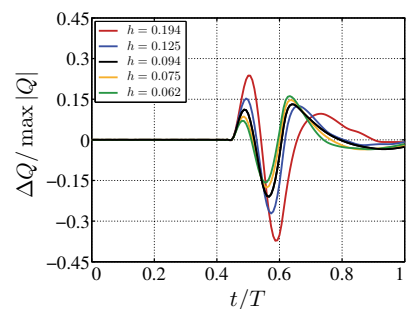

(c)

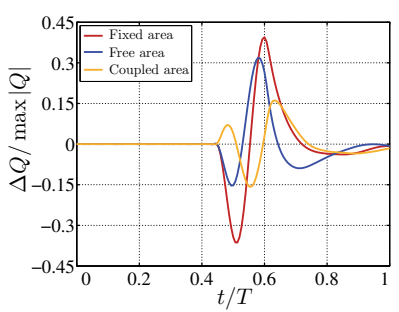

(f)

FIG. 5.3. Volumetric flow rate wave reflection at the coupling interfaces for different values of the fluid mesh size (note that also the solid mesh size changes accordingly). As in Figure 5.2, the black curve corresponds to the reference parameters. (a) Fixed area boundary condition on the 3-D solid ring. (b) Free area boundary condition on the 3-D solid ring. (c) Continuity of the area at the coupling interfaces. (d) $h=0.094$. (e) $h=0.075$. (f) $h=0.062$.

when the 3-D solid ring is fixed. More precisely, the reflection generated by using such a constraining boundary condition is around twice the size of that observed when the other two approaches are employed, motivating the need for such more relaxed conditions on the coupling interface. In contrast, there is no clear advantage in prescribing the continuity of the area of the pipes, with respect to letting the deformation of the 3-D boundary solid ring free. Indeed, such behavior depends upon the specific case. (See Figure 5.2(b) and Figure 5.2(c) for varying Young's modulus.)

Regarding the sensitivity analysis with respect to the main 3-D solid parameters, we observe that the value of the Young's modulus has an impact on the reflection, even if the shape of the wave does not change significantly. More precisely, the vertical difference between the subsequent peaks in the wave is nearly constant such that the amplitude of the reflection is almost the same. In contrast, the Poisson ratio does not have any effect on the reflection. Finally, we observe that modeling the inertia of the 3-D solid wall leads to significant differences only in the presence of the free solid ring boundary condition. More precisely, the reflection amplifies probably due to the additional modeling heterogeneity introduced in this specific case. (We recall that our 1-D FSI model does not account for the inertia of the wall.) By applying the other two solid ring boundary conditions, the displacement of the ring is controlled by the boundary data such that the inertial effects at the boundary are smoothed out.

Last but not least, in Figure 5.3 we study the reflection amplitude as a function of the 3-D mesh size. The first row of images shows opposite behavior between the case where the continuity of the area is imposed and the other two types of boundary conditions. More precisely, a refinement of the mesh leads to a progressive reduction of the reflection amplitude in the former case and an increase in the latter. In the second row of images we compare the reflection amplitude as a function of the interface 


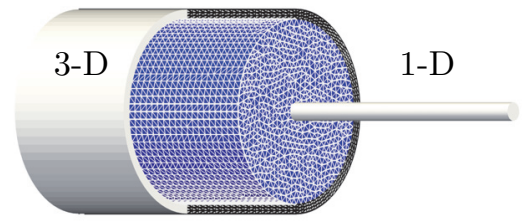

(a)

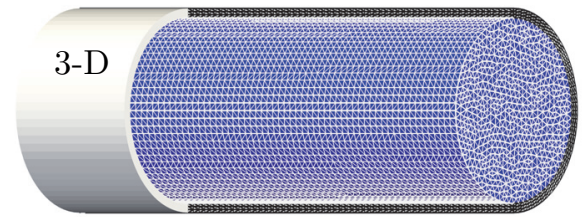

(b)

FIG. 5.4. Schematic representation of the benchmark case. (a) The wave flow propagates from the 3-D FSI model to the 1-D FSI one. The length of each pipe is equal to 3. (b) The reference case: A single 3-D FSI pipe of length 6.

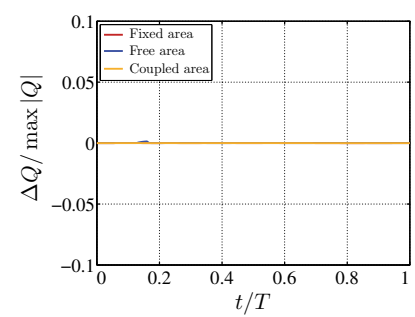

(a)

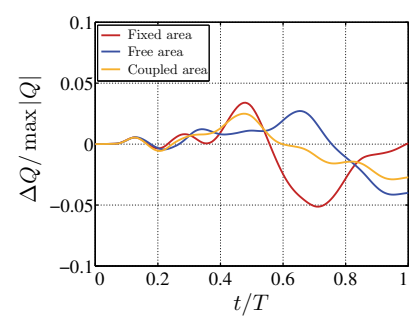

(b)

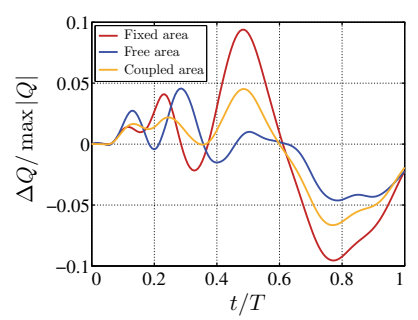

(c)

FIG. 5.5. Volumetric flow rate wave reflection at different locations of the 3-D FSI pipe. (a) Left inlet. (b) Half section. (c) Right outlet (coupling interface).

condition for fixed values of the 3-D mesh size. From the images it is evident that, in contrast with the coarse mesh results, with the finer mesh the reflection amplitude of the case where the continuity of the area is imposed is smaller with respect to that obtained by using the other two interface conditions. By assuming that the result provided by the finer mesh is the most accurate, we might conclude that the continuity of the area represents the most suitable interface condition between the considered heterogeneous models. Nevertheless, further analyses should be performed to confirm this behavior in more general cases. Note also that a similar analysis with respect to the 1-D mesh size does not produce any significant difference. In particular, below a certain mesh size, the results in the 1-D segment are nearly the same.

5.2. From 3-D to 1-D wave flow propagation. In the second case, described in Figure 5.4, we study the reflection amplitude generated at the coupling interfaces when a wave propagates from a 3-D FSI pipe (on the left) to a 1-D FSI segment (on the right); see Figure 5.4(a). As a reference case we consider the solution computed by solving the problem with a single 3-D FSI pipe of length 6 (see Figure 5.4(b)).

Figure 5.5 shows the same quantities as in Figure 5.2, with the inverted configuration. By comparing the reflection amplitude in Figure 5.2 with the results in Figure 5.5(c), we observe that in the latter case the amplitude of the reflection is much smaller, around one-third of the previous case. This can be explained by observing that the 1-D problem is hyperbolic, and at the coupling interface it behaves as a passive element which absorbs the flow generated by the 3-D pipe. By way of contrast, in the previous case, the flow generated by the 1-D segment is passed to a nonhyperbolic model which reflects an active feedback to the connected 1-D segment.

Finally, a further comparison is presented in Figure 5.6, where on the $y$-axis we show the radial scale factor (in percentage units), whose definition is given in (4.5). 


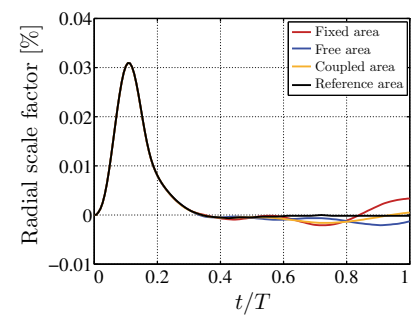

(a)

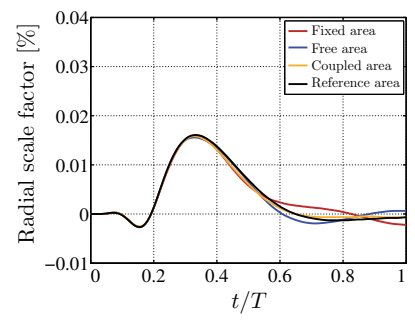

(b)

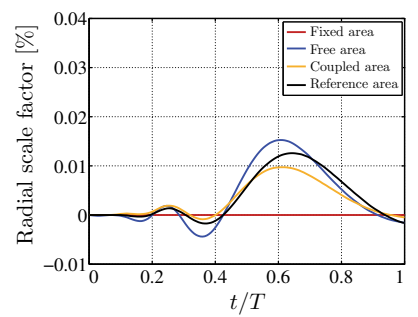

(c)

FIG. 5.6. Radial scale factor of the 3-D FSI pipe in Figure 5.4(a) at different locations. The black line is the reference value (see Figure 5.4(b)). (a) Left inlet. (b) Half section. (c) Right outlet (coupling interface).

This result leads to the same conclusions discussed in section 5.1. In particular, the amplitude of the reflection is always larger when the 3-D solid ring is fixed, while the other two approaches yield better results. Moreover, we notice that prescribing the continuity of the area of the pipes produces a slightly better result than does letting free the deformation of the 3-D boundary solid ring.

6. Computational cost analysis in abdominal aorta simulation. In order to assess the efficiency of the methodology presented here, we compare the number of iterations and the CPU time required to solve a realistic flow simulation as a function of several parameters, i.e., the 3-D FSI time discretization scheme, the number of coupling interfaces, and the algorithm for solving the interface problem. To perform this analysis we select a 3-D geometry of an arterial abdominal aorta, equipped with six coupling interfaces. At each boundary interface we connect a 1-D pipe featuring a length of $1.5 \mathrm{~cm}$ and a uniform reference area equal to that of the nearby fluid interface of the 3-D FSI abdominal aorta. The interface problem is defined by imposing the coupling flow on all six coupling interfaces, while all the boundary rings of the 3-D solid problem are fixed. The problem is finally closed by imposing six periodic volumetric flow rate profiles on the other sides of the 1-D pipes, as shown in Figure 6.1. These data have been taken from the solution of the full 1-D arterial tree described in [31, section 5].

Regarding the wall parameters, we use the same values provided in [31]. On the solid external wall $\Gamma_{\mathrm{S} \text {,ext }}^{0}$ of the 3 -D FSI abdominal aorta we apply a proper Robin boundary condition, which also takes into account the viscoelastic effects of the arterial wall, while we set $\phi_{\mathrm{S}}=10^{\circ}, T_{\mathrm{S}}=0.24 \mathrm{~s}$, and $P_{\text {ext }}=100000 \mathrm{dyn} / \mathrm{cm}^{2}$ for the 1-D FSI pipes. The Poisson ratio $\nu_{\mathrm{S}}$ is 0.5 for the 1-D FSI pipes and 0.475 for the 3-D FSI abdominal aorta. The thickness of the vessel walls has been set equal to $10 \%$ of the local radius. Finally, the other parameters that define the problem are $\rho_{\mathrm{F}}=1.04 \mathrm{~g} / \mathrm{cm}^{3}, \mu_{\mathrm{F}}=0.035 \mathrm{~g} / \mathrm{cm} / \mathrm{s}, \alpha_{\mathrm{F}}=1.1, E_{\mathrm{S}}=3000000 \mathrm{dyn} / \mathrm{cm}^{2}$, and $\rho_{\mathrm{S}}=1.2 \mathrm{~g} / \mathrm{cm}^{3}$.

The imposed tolerance for the interface problem is $10^{-6}$, while that for the $3-\mathrm{D}$ FSI problem is $10^{-7}$. The mesh of the fluid part of the 3-D abdominal aorta consists of 361969 tetrahedral elements with 62741 vertices, while the solid part is made of 236004 tetrahedral elements with 49675 vertices. The corresponding average space discretizations for the fluid and solid problems are $0.026 \mathrm{~cm}$ and $0.044 \mathrm{~cm}$, respectively. Regarding the 1-D FSI segments, we use a uniform space discretization of $0.1 \mathrm{~cm}$, corresponding to 16 vertices. The global time step is set equal to $10^{-3} \mathrm{~s}$. All the 

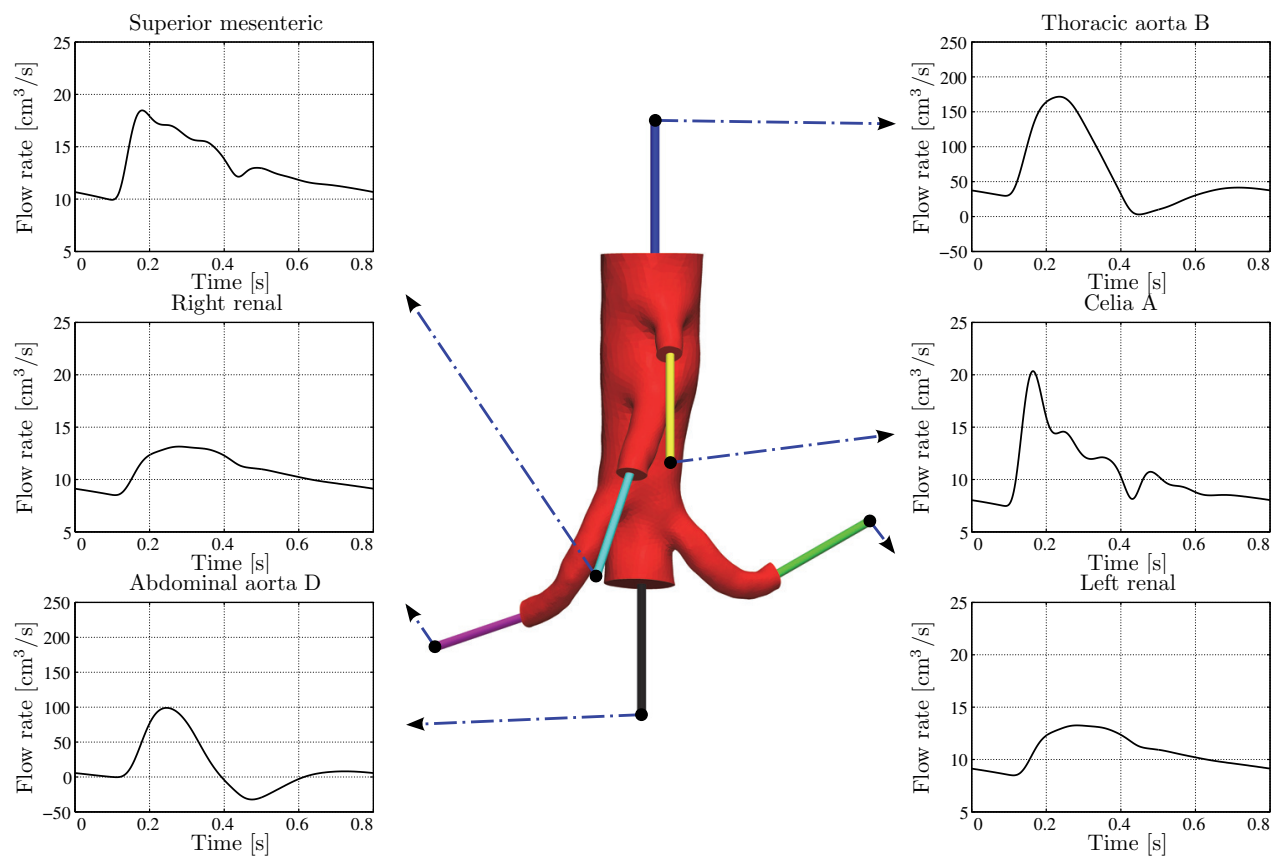

FIG. 6.1. View of the 3-D FSI abdominal aorta (red, center) coupled with six 1-D FSI arterial segments (other colors, rods). On the two sides of the image, the periodic inflow/outflow volumetric flow rate profiles imposed on the six 1-D pipes are shown. More precisely, the flow imposed at the top of the 1-D thoracic aorta segment (top-right figure) is incoming, while the other five flows are outgoing.

simulations were performed on five cluster nodes with two Intel Xeon processors X5550 (quad core, $8 \mathrm{MB}$ cache, $2.66 \mathrm{GHz} \mathrm{CPU}$ ) each, for a total of $40 \mathrm{CPUs}$ interconnected by $20 \mathrm{~Gb} / \mathrm{s}$ InfiniBand architecture.

To set up the comparison we first run three heart beats of the problem described in Figure 6.1, to reach a quasi-periodic solution in the entire network. Then, starting from this initial condition, we run twenty time steps (corresponding to $0.020 \mathrm{~s}$ ) for several configurations. Each of these configurations is set by removing one by one the 1-D segments from the initial problem. More precisely, from the initial configuration in Figure 6.1, the 1-D pipes are removed in the following order: left renal (green, lower-right), right renal (magenta, middle-left), superior mesenteric (cyan, top-left), celiac A (yellow, middle-right), abdominal aorta D (black, lower-left), and, finally, thoracic aorta B (blue, top-right). Note that, to preserve the same physics of the problem, each removed 1-D pipe has been replaced with the corresponding boundary volumetric flow rate profile of the fully coupled problem. Finally, we compute the average number of iterations per time step required for the solution of the interface problem and the CPU time of the entire simulation normalized over the CPU time needed for the reference case, i.e., the 3-D FSI abdominal aorta discretized by a GCE scheme and without any coupled 1-D segment. The results of this comparison are summarized in Tables 6.1 and 6.2 .

From the analysis of these results, we conclude that increasing the number of coupling nodes, which yields an increase of the size of the interface problem, produces a slight variation of the average number of iterations required to solve the coupled problem. A similar consideration holds when comparing the results for the two solu- 
TABLE 6.1

Average number of coupling iterations required for the simulation of the 3-D FSI abdominal aorta coupled with a progressively decreasing number of 1-D segments. The average number of Newton iterations for the convergence of the FI 3-D FSI problem is 3.88.

\begin{tabular}{cccccc}
\hline & \multicolumn{2}{c}{ GCE } & & \multicolumn{2}{c}{ FI } \\
\cline { 2 - 3 } \cline { 5 - 6 } 1-D pipes & Newton & Broyden & & Newton & Broyden \\
\hline 6 & 2.00 & 2.00 & & 7.95 & 9.15 \\
5 & 2.00 & 2.00 & & 7.20 & 9.05 \\
4 & 2.00 & 2.00 & & 6.05 & 8.00 \\
3 & 2.00 & 2.00 & & 6.00 & 7.75 \\
2 & 1.75 & 2.00 & & 6.00 & 7.90 \\
1 & 1.25 & 1.95 & & 5.95 & 7.70 \\
\hline
\end{tabular}

TABLE 6.2

Relative CPU time required for the simulation of the 3-D FSI abdominal aorta coupled with a progressively decreasing number of 1-D segments. In the last two columns, the values in the braces correspond to the same CPU times normalized over the CPU time needed by the FI reference case, which is 5.13 times slower than the GCE one.

\begin{tabular}{cccccc}
\hline & \multicolumn{2}{c}{ GCE } & & \multicolumn{2}{c}{ FI } \\
\cline { 2 - 3 } \cline { 5 - 6 } 1-D pipes & Newton & Broyden & & Newton & Broyden \\
\hline 6 & 9.91 & 2.33 & & $120.20[23.42]$ & $45.46[8.86]$ \\
5 & 8.58 & 2.30 & & $94.09[18.34]$ & $44.93[8.76]$ \\
4 & 7.20 & 2.26 & & $67.77[13.21]$ & $40.22[7.84]$ \\
3 & 6.00 & 2.23 & & $57.08[11.13]$ & $39.04[7.61]$ \\
2 & 4.29 & 2.20 & & $47.99[9.35]$ & $39.64[7.73]$ \\
1 & 2.27 & 2.15 & & $36.33[7.08]$ & $38.65[7.53]$ \\
\hline
\end{tabular}

tion algorithms. More precisely, the Newton method behaves as the Broyden one in the case of a GCE time discretization, and slightly better than the Broyden method in the FI approach. However, there is a visible increase in the average number of iterations between the GCE and the FI time discretization schemes. This is due to the strong nonlinearities of the FI approach.

Regarding CPU time, we observe quite different behavior. In particular, the Newton method shows a strong dependence on the number of coupling nodes of the problem. This is justified by the fact that, in this example, each coupling node requires the computation of two additional Jacobian entries, which in turn requires the additional solution of a 3-D FSI tangent problem and of a 1-D FSI model (to perform the finite difference approximation), at each nonlinear Richardson iteration. In contrast, the CPU time required to solve the interface problem with the Broyden method is almost the same. More precisely, by using the Broyden method the CPU time required to solve the coupled problem is about 2.3 and 5 times that required to solve the uncoupled 3-D FSI model discretized with the GCE and FI time discretization approaches, respectively. Last but not least, it is interesting to notice that there is an increase of more than one order of magnitude in the CPU time required for the solution of the coupled problem when using the FI approach with respect to the GCE case.

In view of these considerations, it is clear that from the computational viewpoint the Broyden method performs much better than the Newton method. In addition, the use of a GCE time discretization is recommended, unless other needs would support the use of the more expensive FI approach. Further comparisons between these two approaches, with a focus on the main physical quantities, are addressed in [10]. 
7. Conclusions. In this work, we presented a numerical technique for the coupling of 1-D and 3-D FSI models for arterial networks. A full description of the FSI model equations has been provided, together with the strategies used to solve the numerical problems.

The global network of elements is solved by using a partitioned approach, where the models communicate only through the exchange of averaged/integrated quantities across the boundary interfaces. In particular, the fluid coupling equations are based on the conservation of the volumetric flow rate and on the continuity of the average of the normal component of the traction vector. Regarding the solid part of the problems, since the 1-D FSI model is already closed by the fluid coupling equations, we mainly focus on the 3-D FSI one. More precisely, we devise three different sets of boundary conditions to close the 3-D solid problem. All of them are stand-alone boundary conditions, that can be employed with and without connected 1-D models. Moreover the third one can be further extended to account for the continuity of the vessel area between the 3-D and 1-D FSI problems.

The problem at the coupling interfaces is solved by a classical nonlinear Richardson approach. In particular, two different numerical strategies have been selected: the Newton/inexact-Newton and the Broyden methods. The former requires the computation/approximation of the Jacobian matrix: for the 3-D FSI model this is done by solving the associated tangent problem, while for the 1-D FSI problem a finite difference approximation scheme is used. Regarding the Broyden method, it is based just on the evaluation of the residual, leading to a very cheap formulation. Note, however, that the Broyden matrix needs to be initialized with a good approximation of the Jacobian, which can be retrieved by using the former method.

The methodology presented here has been tested on several cases, ranging from simple benchmark examples, consisting of serial connection of pipes, to a more physiological example of the arterial network. The former case has been used to carry out a sensitivity analysis of the spurious backward reflections that might be generated at the coupling interfaces between the dimensionally heterogeneous models. The latter served to set up a comparison in terms of number of iterations and CPU time between the different solution algorithms; indeed, it also proved the robustness of the methodology devised here under physiological conditions.

The study presented in this work can be further extended, for instance, by performing a sensitivity analysis with respect to other parameters, such as the viscoelastic coefficient of the 1-D FSI model. However, the results are expected to be similar to those presented in this work. Moreover, we remark that viscoelastic effects can be included in the 3-D FSI model by considering, e.g., a Robin boundary condition on the external wall. Indeed, the sensitivity analysis presented here showed that the spurious reflections which appear when coupling dimensionally heterogeneous FSI problems are mainly due to the heterogeneous nature of the models equations rather than to possible physical inconsistencies.

\section{REFERENCES}

[1] S. Badia, F. Nobile, And C. Vergara, Fluid-structure partitioned procedures based on Robin transmission conditions, J. Comput. Phys., 227 (2008), pp. 7027-7051.

[2] Y. Bazilevs, V. M. Calo, T. J. R. Hughes, and Y. Zhang, Isogeometric fluid-structure interaction: Theory, algorithms, and computations, Comput. Mech., 43 (2008), pp. 3-37.

[3] P. J. Blanco, R. A. Feijóo, and S. A. Urquiza, A unified variational approach for coupling $3 D-1 D$ models and its blood flow applications, Comput. Methods Appl. Mech. Engrg., 196 (2007), pp. 4391-4410. 
[4] P. J. Blanco, J. S. Leiva, R. A. Feijóo, And G. C. Buscaglia, Black-box decomposition approach for computational hemodynamics: One-dimensional models, Comput. Methods Appl. Mech. Engrg., 200 (2011), pp. 1389-1405.

[5] P. J. Blanco, S. A. Urquiza, and R. A. Feisóo, Assessing the influence of heart rate in local hemodynamics through coupled 3D-1D-0D models, Int. J. Numer. Methods Biomed. Engrg., 26 (2010), pp. 890-903.

[6] J. Bonnemain, A. C. I. Malossi, M. Lesinigo, S. Deparis, A. Quarteroni, and L. K. von SEGESSER, Numerical simulation of left ventricular assist device implantations: Comparing the ascending and the descending aorta cannulations, Med. Eng. Phys., (2013), to appear.

[7] E. Burman, M. A. Fernández, and P. Hansbo, Continuous interior penalty finite element method for Oseen's equations, SIAM J. Numer. Anal., 44 (2006), pp. 1248-1274.

[8] P. Causin, J.-F. Gerbeau, and F. Nobile, Added-mass effect in the design of partitioned algorithms for fluid-structure problems, Comput. Methods Appl. Mech. Engrg., 194 (2005), pp. $4506-4527$.

[9] M. Cinthio, A. R. Ahlgren, J. Bergkvist, T. Jansson, H. W. Persson, And K. Lindström, Longitudinal movements and resulting shear strain of the arterial wall, Am. J. Physiol. Heart Circ. Physiol., 291 (2006), pp. H394-H402.

[10] P. Crosetto, Fluid-Structure Interaction Problems in Hemodynamics: Parallel Solvers, Preconditioners, and Applications, Ph.D. thesis, École Polytechnique Fédérale de Lausanne, Lausanne, Switzerland, 2011.

[11] P. Crosetto, S. Deparis, G. Fourestey, and A. Quarteroni, Parallel algorithms for fluid-structure interaction problems in haemodynamics, SIAM J. Sci. Comput., 33 (2011), pp. 1598-1622.

[12] S. Deparis, M. Discacciati, G. Fourestey, and A. Quarteroni, Fluid-structure algorithm based on Steklov-Poincaré operators, Comput. Methods Appl. Mech. Engrg., 195 (2006), pp. $5797-5812$.

[13] S. Deparis, M. Discacciati, and A. Quarteroni, A domain decomposition framework for fluid-structure interaction problems, in Proceedings of the Third International Conference on Computational Fluid Dynamics (ICCFD3), Springer, Berlin, Heidelberg, 2006, pp. 4158.

[14] M. A. Fernández And M. Moubachir, A Newton method using exact Jacobian for solving fluid-structure coupling, Comput. \& Structures, 83 (2005), pp. 127-142.

[15] L. Formaggia, J.-F. Gerbeau, F. Nobile, and A. Quarteroni, On the coupling of $3 D$ and $1 D$ Navier-Stokes equations for flow problems in compliant vessels, Comput. Methods Appl. Mech. Engrg., 191 (2001), pp. 561-582.

[16] L. Formaggia, J.-F. Gerbeau, F. Nobile, and A. Quarteroni, Numerical treatment of defective boundary conditions for the Navier-Stokes equations, SIAM J. Numer. Anal., 40 (2002), pp. 376-401.

[17] L. Formaggia, D. Lamponi, And A. Quarteroni, One-dimensional models for blood flow in arteries, J. Engrg. Math., 47 (2003), pp. 251-276.

[18] L. Formaggia, D. Lamponi, M. Tuveri, and A. Veneziani, Numerical modeling of $1 D$ arterial networks coupled with a lumped parameters description of the heart, Comput. Methods Biomech. Biomed. Eng., 9 (2006), pp. 273-288.

[19] L. Formaggia, A. Moura, And F. Nobile, On the stability of the coupling of $3 D$ and $1 D$ fluid-structure interaction models for blood flow simulations, ESAIM Math. Model. Numer. Anal., 41 (2007), pp. 743-769.

[20] L. Formaggia And F. Nobile, A stability analysis for the arbitrary Lagrangian Eulerian formulation with finite elements, East-West J. Numer. Math., 7 (1999), pp. 105-131.

[21] L. Formaggia, A. Quarteroni, and A. Veneziani, Cardiovascular Mathematics, Model. Simul. Appl. 1, Springer-Verlag, Milan, 2009.

[22] L. Formaggia, A. Quarteroni, and C. Vergara, On the physical consistency between threedimensional and one-dimensional models in haemodynamics, J. Comput. Phys., (2012), to appear.

[23] L. Gerardo-Giorda, F. Nobile, and C. Vergara, Analysis and optimization of Robin-Robin partitioned procedures in fluid-structure interaction problems, SIAM J. Numer. Anal., 48 (2010), pp. 2091-2116.

[24] L. Grinberg and G. E. Karniadakis, Outflow boundary conditions for arterial networks with multiple outlets, Ann. Biomed. Eng., 36 (2008), pp. 1496-1514.

[25] J. G. Heywood, R. Rannacher, and S. Turek, Artificial boundaries and flux and pressure conditions for the incompressible Navier-Stokes equations, Int. J. Numer. Methods Fluids, 22 (1996), pp. 325-352. 
[26] H. J. Kim, I. E. Vignon-Clementel, C. A. Figueroa, J. F. LaDisa, K. E. Jansen, J. A. FEINSTEIN, AND C. A. TAYLOR, On coupling a lumped parameter heart model and a threedimensional finite element aorta model, Ann. Biomed. Eng., 37 (2009), pp. 2153-2169.

[27] U. Küttler, M. Gee, C. Förster, A. Comerford, and W. A. Wall, Coupling strategies for biomedical fluid-structure interaction problems, Int. J. Numer. Methods Biomed. Engrg., 26 (2010), pp. 305-321.

[28] J. S. Leiva, P. J. Blanco, and G. C. Buscaglia, Partitioned analysis for dimensionallyheterogeneous hydraulic networks, Multiscale Model. Simul., 9 (2011), pp. 872-903.

[29] Life V Project, parallel finite element library, http://www.lifev.org.

[30] A. C. I. MAlossi, Partitioned Solution of Geometrical Multiscale Problems for the Cardiovascular System: Models, Algorithms, and Applications, Ph.D. thesis, École Polytechnique Fédérale de Lausanne, Lausanne, Switzerland, 2012.

[31] A. C. I. Malossi, P. J. Blanco, and S. Deparis, A two-level time step technique for the partitioned solution of one-dimensional arterial networks, Comput. Methods Appl. Mech. Engrg., 237-240 (2012), pp. 212-226.

[32] A. C. I. Malossi, P. J. Blanco, S. Deparis, and A. Quarteroni, Algorithms for the partitioned solution of weakly coupled fluid models for cardiovascular flows, Int. J. Numer. Methods Biomed. Engrg., 27 (2011), pp. 2035-2057.

[33] H. G. Matthies And J. Steindorf, Partitioned strong coupling algorithms for fluid-structure interaction, Comput. \& Structures, 81 (2003), pp. 805-812.

[34] P. Moireau, N. Xiao, M. Astorino, C. A. Figueroa, D. Chapelle, C.-A. Taylor, and J.-F. Gerbeau, External tissue support and fluid-structure simulation in blood flows, Biomech. Model. Mechanobiol., 11 (2012), pp. 1-18.

[35] F. Nobile And C. Vergara, An effective fluid-structure interaction formulation for vascular dynamics by generalized Robin conditions, SIAM J. Sci. Comput., 30 (2008), pp. 731-763.

[36] J. T. Ottesen, M. S. Olufsen, and J. K. Larsen, Applied Mathematical Models in Human Physiology, Monographs on Mathematical Modeling and Computation MM09, SIAM, Philadelphia, 2004.

[37] T. Passerini, M. de Luca, L. Formaggia, A. Quarteroni, and A. Veneziani, A 3D/1D geometrical multiscale model of cerebral vasculature, J. Engrg. Math., 64 (2009), pp. 319330.

[38] L. Quartapelle, Numerical Solution of the Incompressible Navier-Stokes Equations, Internat. Ser. Numer. Math. 113, Birkhäuser-Verlag, Basel, 1993.

[39] A. Quarteroni And L. Formaggia, Mathematical modelling and numerical simulation of the cardiovascular system, in Modelling of Living Systems, Handbook Numer. Anal. Ser., Elsevier, New York, 2003.

[40] A. Quarteroni and A. Veneziani, Analysis of a geometrical multiscale model based on the coupling of ODEs and PDEs for blood flow simulations, Multiscale Model. Simul., 1 (2003), pp. 173-195.

[41] P. Reymond, F. Merenda, F. Perren, D. Rüfenacht, and N. Stergiopulos, Validation of a one-dimensional model of the systemic arterial tree, Am. J. Physiol. Heart Circ. Physiol., 297 (2009), pp. H208-H222.

[42] S. J. Sherwin, V. Franke, J. Peiró, And K. Parker, One-dimensional modelling of a vascular network in space-time variables, J. Engrg. Math., 47 (2003), pp. 217-250.

[43] T. E. Tezduyar, S. Sathe, and K. Stein, Solution techniques for the fully discretized equations in computation of fluid-structure interactions with the space-time formulations, Comput. Methods Appl. Mech. Engrg., 195 (2006), pp. 5743-5753.

[44] I. E. Vignon-Clementel, C. A. Figueroa, K. E. Jansen, and C. A. Taylor, Outflow boundary conditions for three-dimensional finite element modeling of blood flow and pressure in arteries, Comput. Methods Appl. Mech. Engrg., 195 (2006), pp. 3776-3796. 